\title{
Homeomorphisms of the Mandelbrot Set
}

\author{
Wolf Jung
}

\begin{abstract}
On subsets of the Mandelbrot set, $\mathcal{E}_{M} \subset \mathcal{M}$, homeomorphisms are constructed by quasi-conformal surgery. When the dynamics of quadratic polynomials is changed piecewise by a combinatorial construction, a general theorem yields the corresponding homeomorphism $h: \mathcal{E}_{M} \rightarrow \mathcal{E}_{M}$ in the parameter plane. Each $h$ has two fixed points in $\mathcal{E}_{M}$, and a countable family of mutually homeomorphic fundamental domains. Possible generalizations to other families of polynomials or rational mappings are discussed.

The homeomorphisms on subsets $\mathcal{E}_{M} \subset \mathcal{M}$ constructed by surgery are extended to homeomorphisms of $\mathcal{M}$, and employed to study groups of nontrivial homeomorphisms $h: \mathcal{M} \rightarrow \mathcal{M}$. It is shown that these groups have the cardinality of $\mathbb{R}$, and they are not compact.
\end{abstract}

Preprint of a paper submitted to Dynamics in the Complex Plane, proceedings of a symposium in honour of Bodil Branner, June 19-21 2003, Holbæk.

\section{Introduction}

Consider the family of complex quadratic polynomials $f_{c}(z):=z^{2}+c$. They are parametrized by $c \in \mathbb{C}$, which is at the same time the critical value of $f_{c}$, since 0 is the critical point. The filled Julia set $\mathcal{K}_{c}$ of $f_{c}$ is a compact subset of the dynamic plane. It contains all $z \in \mathbb{C}$ which are not attracted to $\infty$ under the iteration of $f_{c}$, i.e. $f_{c}^{n}(z) \nrightarrow \infty$. The global dynamics is determined qualitatively by the behavior of the critical point or critical value under iteration. E.g., by a classical theorem of Fatou, $\mathcal{K}_{c}$ is connected iff $f_{c}^{n}(c) \nrightarrow \infty$, i.e. $c \in \mathcal{K}_{c}$. The Mandelbrot set $\mathcal{M}$ is a subset of the parameter plane, it contains precisely the parameters with this property. Although it can be defined by the recursive computation of the critical orbit, with no reference to the whole dynamic plane, most results on $\mathcal{M}$ are obtained by an interplay between both planes: starting with a subset $\mathcal{E}_{M} \subset \mathcal{M}$, employ the dynamics of $f_{c}$ to find a common structure in $\mathcal{K}_{c}$ for all $c \in \mathcal{E}_{M}$. Then an analogous structure will be found in $\mathcal{E}_{M}$, i.e. in the parameter plane. This principle has various precise formulations. Most important is its application to external rays: these are curves in the complement of $\mathcal{K}_{c}$ (dynamic rays) or in the complement of $\mathcal{M}$ (parameter rays), which are labeled by an angle $\theta \in S^{1}=\mathbb{R} / \mathbb{Z}$. For rational angles $\theta \in \mathbb{Q} / \mathbb{Z}$, 
these rays are landing at special points in $\partial \mathcal{K}_{c}$ or $\partial \mathcal{M}$, respectively. See Sect. 2.1 for details. When rays are landing together, the landing point is called a pinching point. It can be used to disconnect $\mathcal{K}_{c}$ or $\mathcal{M}$ into well-defined components. The structure of $\mathcal{K}_{c}$, as described by these pinching points, can be understood dynamically, and then these results are transfered to the parameter plane, to understand the structure of $\mathcal{M}$.

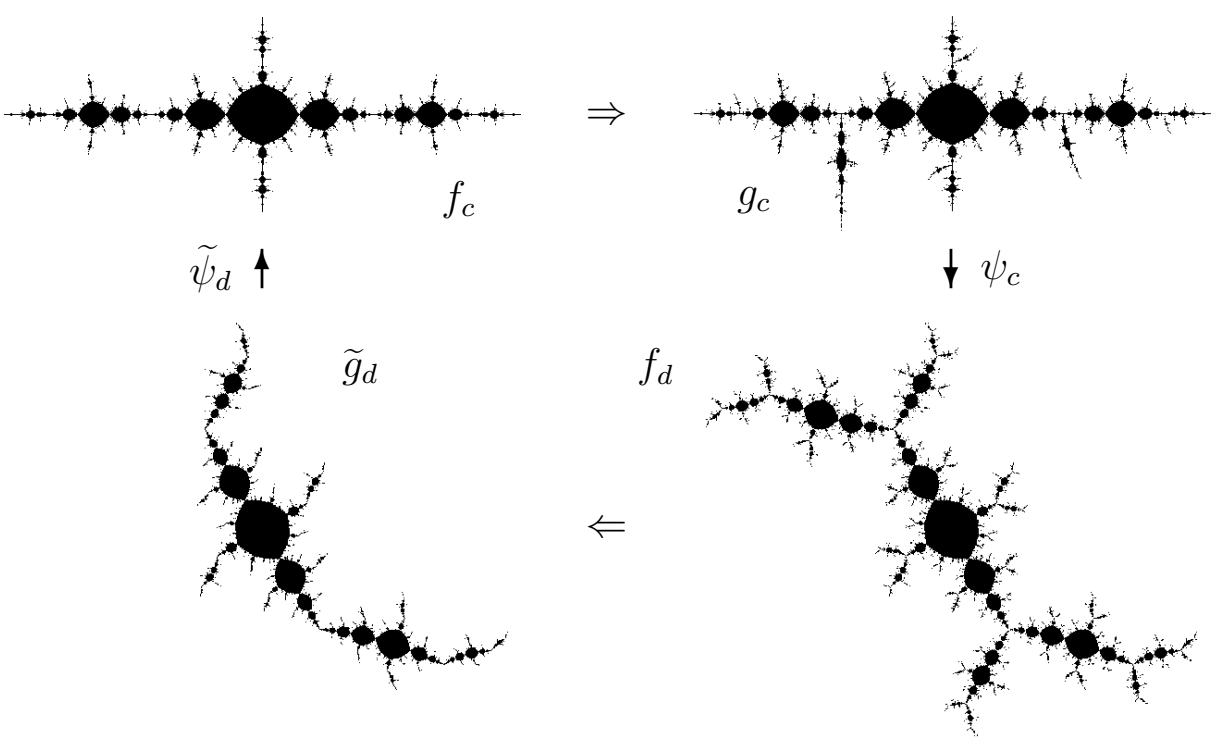

Figure 1: A simulation of Branner-Douady surgery $\Phi_{A}: \mathcal{M}_{1 / 2} \rightarrow \mathcal{T} \subset \mathcal{M}_{1 / 3}$, as explained in the text below. In this simulation, $g_{c}$ and $\widetilde{g}_{d}$ are defined piecewise explicitly, and the required Riemann mappings are replaced with simple affine mappings.

Each filled Julia set $\mathcal{K}_{c}$ is completely invariant under the corresponding mapping $f_{c}$, and this fact explains the self-similarity of these sets. On the other hand, when the parameter $c$ moves through the Mandelbrot set, the corresponding Julia sets undergo an infinite number of bifurcations. By the above principle, the local structure of $\mathcal{M}$ is undergoing corresponding changes as well. But these changes may combine in such a way, that subsets of $\mathcal{M}$ are mutually homeomorphic. Such homeomorphisms can be constructed by quasi-conformal surgery. There are three basic ideas (the first and second apply to more general situations [5]):

- A mapping $g$ with desired dynamics is constructed piecewise, i.e. by piecing together different mappings or different iterates of one mapping. The pieces are defined e.g. by dynamic rays landing at pinching points of the Julia set.

- $g$ cannot be analytic, but one constructs a quasi-conformal mapping $\psi$ such that the composition $f=\psi \circ g \circ \psi^{-1}$ is analytic. This is possible, when a field of infinitesimal ellipses is found that is invariant under $g$. Then $\psi$ is constructed such that it is mapping these ellipses to infinitesimal circles. (See Sect. 2.2 for the precise definition of quasi-conformal mappings.) 
- Suppose that $f_{c}$ is a one-parameter family of analytic mappings, e.g. our quadratic polynomials, and that $g_{c}$ is constructed piecewise from iterates of $f_{c}$ for parameters $c \in \mathcal{E}_{M} \subset \mathcal{M}$. If $\psi_{c} \circ g_{c} \circ \psi_{c}^{-1}=f_{d}$, a mapping in parameter space is obtained from $h(c):=d$. There are techniques to show that $h$ is a homeomorphism.

Homeomorphisms of the Mandelbrot set have been obtained in $[6,1,2,3,13,8]$. We shall discuss the example of the Branner-Douady homeomorphism $\Phi_{A}$, cf. Fig. 1: parameters $c$ in the limb $\mathcal{M}_{1 / 2}$ of $\mathcal{M}$ are characterized by the fact, that the Julia set $\mathcal{K}_{c}$ has two branches at the fixed point $\alpha_{c}$ and at its countable family of preimages. By a piecewise construction, $f_{c}$ is replaced with a new mapping $g_{c}$, such that a third branch appears at $\alpha_{c}$, and thus at its preimages as well. This can be done by cut- and paste techniques on a Riemann surface, or by conformal mappings between sectors in the dynamic plane. Since $g_{c}$ is analytic except in some smaller sectors, it is possible to construct an invariant ellipse field. The corresponding quasi-conformal mapping $\psi_{c}$ is used to conjugate $g_{c}$ to a (unique) quadratic polynomial $f_{d}$, and the mapping in parameter space is defined by $\Phi_{A}(c):=d$. Now the Julia sets of $f_{d}$ and $g_{c}$ are homeomorphic, and the dynamics are conjugate. The parameter $d$ belongs to the limb $\mathcal{M}_{1 / 3}$, since the three branches of $\mathcal{K}_{d}$ at $\alpha_{d}$ are permuted by $f_{d}$ with rotation number $1 / 3$. Now there is an analogous construction of a mapping $\widetilde{g}_{d}$ for $d \in \mathcal{T} \subset \mathcal{M}_{1 / 3}$, which turns out to yield the inverse mapping $\widetilde{\Phi}_{A}$. The Julia set of $\widetilde{g}_{d}$ has lost some arms, and $\widetilde{g}_{d}$ is conjugate to a quadratic polynomial $f_{e}$ again. By showing that $f_{c}$ and $f_{e}$ are conjugate, it follows that $e=c$, thus $\widetilde{\Phi}_{A} \circ \Phi_{A}=\mathrm{id}$. (The uniqueness follows from the fact that these quasi-conformal conjugations are hybrid-equivalences, i.e. conformal almost everywhere on the filled Julia sets [6].)

For the homeomorphisms constructed in this paper, the mapping $g_{c}$ is defined piecewise by compositions of iterates of $f_{c}$, and no cut- and paste techniques or conformal mappings are used. Then the Julia sets of $f_{c}$ and $g_{c}$ are the same, and no arms are lost or added in the parameter plane either: a subset $\mathcal{E}_{M} \subset \mathcal{M}$ is defined by disconnecting $\mathcal{M}$ at two pinching points, and this subset is mapped onto itself by the homeomorphism (which is not the identity, of course). Thus a countable family of mutually homeomorphic subsets is obtained from one construction. General combinatorial assumptions are presented in Sect. 3.1, which allow the definition of a preliminary mapping $g_{c}^{(1)}$ analogous to the example in Fig. 2: it differs from $f_{c}$ on two strips $V_{c}, W_{c}$, where it is of the form $f_{c}^{-n} \circ\left( \pm f_{c}^{m}\right)$. Basically, we only need to find four strips with $\overline{V_{c} \cup W_{c}}=\overline{\widetilde{V}_{c} \cup \widetilde{W}_{c}}$, such that these are mapped as $V_{c} \rightarrow \widetilde{V}_{c}$, $W_{c} \rightarrow \widetilde{W}_{c}$ by suitable compositions of $\pm f_{c}^{ \pm 1}$.

\section{Theorem 1.1 (Construction and Properties of $h$ )}

1. Given the combinatorial construction of $\mathcal{E}_{M} \subset \mathcal{M}$ and $g_{c}^{(1)}$ for $c \in \mathcal{E}_{M}$ according to Def. 3.1, there is a family of "quasi-quadratic" mappings $g_{c}$ coinciding with $g_{c}^{(1)}$ on the filled Julia sets $\mathcal{K}_{c}$. These are hybrid-equivalent to unique quadratic polynomials.

2. The mapping $h: \mathcal{E}_{M} \rightarrow \mathcal{E}_{M}$ in parameter space is defined as follows: for $c \in \mathcal{E}_{M}$, 
find the polynomial $f_{d}$ that is hybrid-equivalent to $g_{c}$, and set $h(c):=d$. It does not depend on the precise choice of $g_{c}$ (only on the combinatorial definition of $g_{c}^{(1)}$ ). Now $h$ is a homeomorphism, and analytic in the interior of $\mathcal{E}_{M}$.

3. $h$ is a non-trivial homeomorphism of $\mathcal{E}_{M}$ onto itself, fixing the vertices a and $b$. $h$ and $h^{-1}$ are Hölder continuous at Misiurewicz points and Lipschitz continuous at $a$ and $b$. Moreover, $h$ is expanding at $a$ and contracting at b, cf. Fig. 3: for $c \in \mathcal{E}_{M} \backslash\{a, b\}$ we have $h^{n}(c) \rightarrow b$ as $n \rightarrow \infty$, and $h^{-n}(c) \rightarrow a$. There is a countable family of mutually homeomorphic fundamental domains.

4. $h$ extends to a homeomorphism between strips, $h: \mathcal{P}_{M} \rightarrow \widetilde{\mathcal{P}}_{M}$, which is quasiconformal in the exterior of $\mathcal{M}$.

The power of Thm. 1.1 lies in turning combinatorial data into homeomorphisms. The creative step remaining is to find eight angles $\Theta_{i}^{ \pm}$, such that there are compositions of $\pm f_{c}^{ \pm 1}$ mapping $V_{c} \rightarrow \widetilde{V}_{c}$ and $W_{c} \rightarrow \widetilde{W}_{c}$. When this is done, the existence of a corresponding homeomorphisms is guaranteed by the theorem.
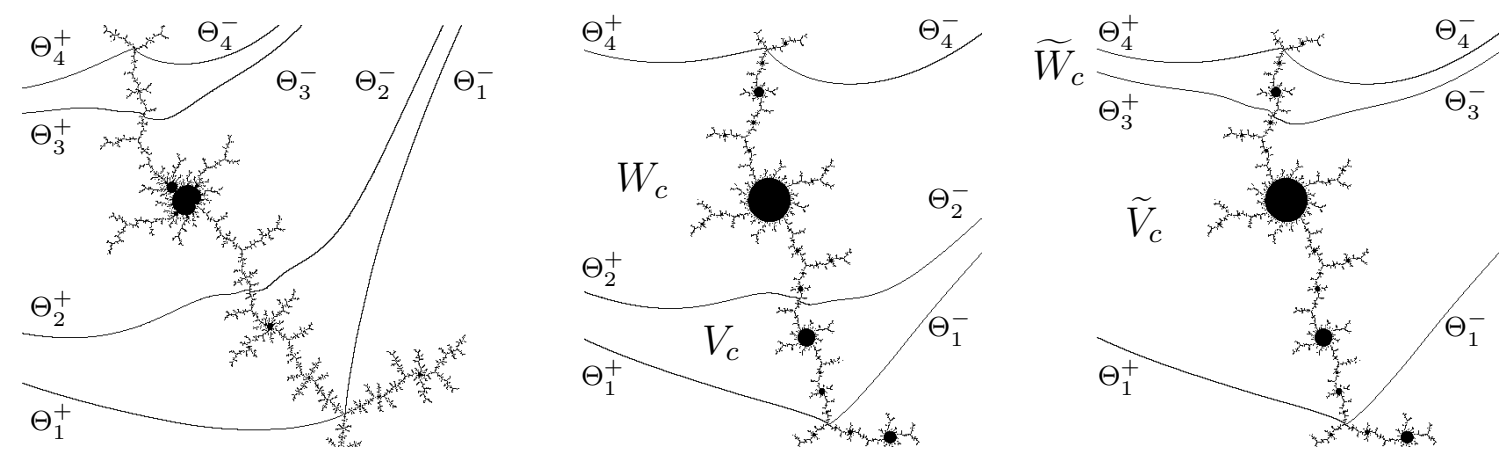

Figure 2: Left: a parameter edge $\mathcal{E}_{M} \subset \mathcal{M}$ and the strip $\mathcal{P}_{M}$. Middle and right: the dynamic edge $\mathcal{E}_{c} \subset \mathcal{K}_{c}$ in the strip $\overline{V_{c} \cup W_{c}}=\overline{\widetilde{V}_{c} \cup \widetilde{W}_{c}}$. According to Sect. 3.1, these strips are bounded by external rays, which belong to eight angles $\Theta_{i}^{ \pm}$. In this example, we have $\Theta_{1}^{-}=11 / 56, \Theta_{2}^{-}=199 / 1008, \Theta_{3}^{-}=103 / 504, \Theta_{4}^{-}=23 / 112, \Theta_{4}^{+}=29 / 112, \Theta_{3}^{+}=131 / 504$, $\Theta_{2}^{+}=269 / 1008$, and $\Theta_{1}^{+}=15 / 56$. The first-return numbers are $k_{w}=\widetilde{k}_{v}=4, k_{v}=\widetilde{k}_{w}=7$. Now $g_{c}=g_{c}^{(1)}$ on $\mathcal{K}_{c}$ and $g_{c}^{(1)}=f_{c} \circ \eta_{c}$, with $\eta_{c}=f_{c}^{-2} \circ\left(-f_{c}^{5}\right)=f_{c}^{-3} \circ\left(+f_{c}^{6}\right): V_{c} \rightarrow \widetilde{V}_{c}$, $\eta_{c}=f_{c}^{-6} \circ\left(-f_{c}^{3}\right): W_{c} \rightarrow \widetilde{W}_{c}$. See also Fig. 3 .

In Sections 2 and 3, basic properties of $\mathcal{M}$ and of quasi-conformal mappings are recalled, and the proof of Thm. 1.1 is sketched by constructing $g_{c}$ and $h$. Related results from the author's thesis [8] are summarized in Sect. 4. These include more examples of homeomorphisms, constructed at chosen Misiurewicz points or on "edges," and the combinatorial description of homeomorphisms. When a oneparameter family of polynomials is defined by critical relations, homeomorphisms in parameter space can be obtained by analogous techniques. The same applies e.g. to the rational mappings arising in Newton's method for cubic polynomials.

H. Kriete has suggested that the homeomorphisms $h: \mathcal{E}_{M} \rightarrow \mathcal{E}_{M}$ constructed by this kind of surgery extend to homeomorphisms of $\mathcal{M}$. Thus they can be used to study 
the homeomorphism group of $\mathcal{M}$, answering a question by K. Keller. In Sect. 5, some possible definitions for groups of non-trivial homeomorphisms are discussed, and some properties are obtained by combining two tools: the characterization of homeomorphisms by permutations of hyperbolic components, and the composition of homeomorphisms constructed by surgery. The groups are not compact, and the groups of non-trivial homeomorphisms have the cardinality of $\mathbb{R}$.
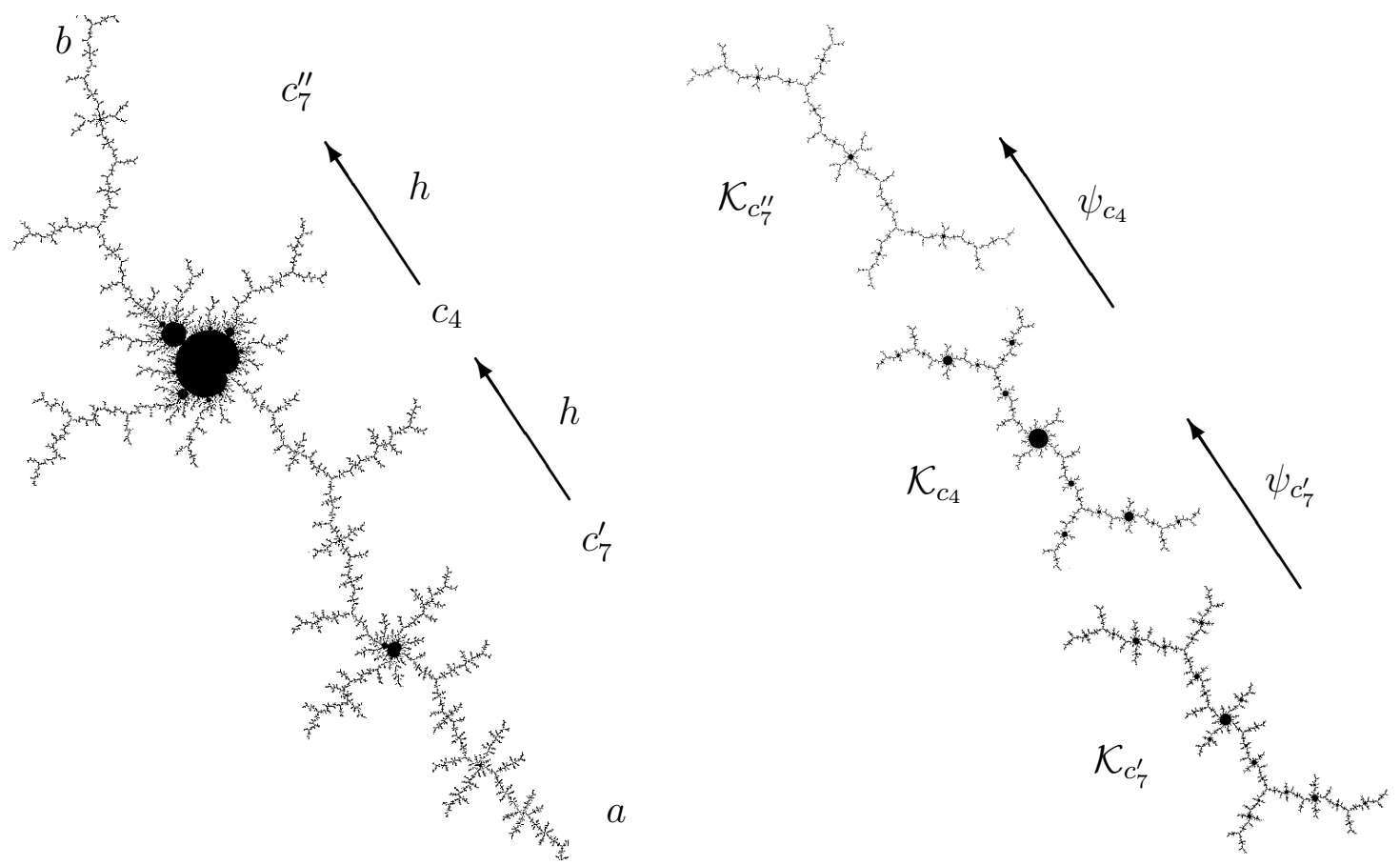

Figure 3: Left: the parameter edge $\mathcal{E}_{M}$ from $a:=\gamma_{M}(11 / 56)$ to $b:=\gamma_{M}(23 / 112)$, the same as in Fig. 2. The homeomorphism $h: \mathcal{E}_{M} \rightarrow \mathcal{E}_{M}$ is expanding at $a$ and contracting at $b$. The centers of periods 4 and 7 are mapped as $h: c_{7}^{\prime} \mapsto c_{4} \mapsto c_{7}^{\prime \prime}$. Right: the filled Julia sets for $c_{7}^{\prime}, c_{4}$, and $c_{7}^{\prime \prime}$ are quasi-conformally homeomorphic. $\left(\mathcal{E}_{c} \subset \mathcal{K}_{c}\right.$ is barely visible in the top right corner.)

\section{Acknowledgment}

Many people have contributed to this work by inspiring discussions and helpful suggestions. I wish to thank in particular Mohamed Barakat, Walter Bergweiler, Volker Enss, Núria Fagella, John Hamal Hubbard, Gerhard Jank, Karsten Keller, Hartje Kriete, Fernando Lledó, Olaf Post, Johannes Riedl, and Dierk Schleicher.

I am especially happy to contribute this paper to the proceedings of a conference in honor of Bodil Branner, since I have learned surgery from her papers [1, 2]. 


\section{Background}

Our main tools are the landing properties of external rays, and sending an ellipse field to circles by a quasi-conformal mapping.

\subsection{The Mandelbrot Set}

$f_{c}(z)=z^{2}+c$ has a superattracting fixed point at $\infty \in \widehat{\mathbb{C}}:=\mathbb{C} \cup\{\infty\}$. The unique Boettcher conjugation is conjugating $f_{c}$ to $F(z):=z^{2}, \Phi_{c} \circ f_{c}=F \circ \Phi_{c}$ in a neighborhood of $\infty$. If the critical point 0 , or the critical value $c$, does not escape to $\infty$, then $\mathcal{K}_{c}$ is connected [5], and the parameter $c$ belongs to the Mandelbrot set $\mathcal{M}$ by definition. Then $\Phi_{c}$ extends to a conformal mapping $\Phi_{c}: \widehat{\mathbb{C}} \backslash \mathcal{K}_{c} \rightarrow \widehat{\mathbb{C}} \backslash \overline{\mathbb{D}}$, where $\overline{\mathbb{D}}$ is the closed unit disk. Dynamic rays $\mathcal{R}_{c}(\theta)$ are defined as preimages of straight rays $\mathcal{R}(\theta)=\{z|1<| z \mid<\infty, \arg (z)=2 \pi \theta\}$ under $\Phi_{c}$. Theses curves in the complement of $\mathcal{K}_{c}$ may land at a point in $\partial \mathcal{K}_{c}$, or accumulate at the boundary without landing. But they are always landing when $\theta$ is rational. Each rational angle $\theta$ is periodic or preperiodic under doubling $(\bmod 1)$. In the former case, the dynamic ray $\mathcal{R}_{c}(\theta)$ is landing at a periodic point $z=\gamma_{c}(\theta) \in \partial \mathcal{K}_{c}$, and at a preperiodic point in the latter case. These properties are understood from $f_{c}\left(\mathcal{R}_{c}(\theta)\right)=\mathcal{R}_{c}(2 \theta)$, since $\arg (F(z))=2 \arg (z)$.

The Mandelbrot set is compact, connected, and full, and the conformal mapping $\Phi_{M}: \widehat{\mathbb{C}} \backslash \mathcal{M} \rightarrow \widehat{\mathbb{C}} \backslash \overline{\mathbb{D}}$ is given by $\Phi_{M}(c):=\Phi_{c}(c)$. (When $c \notin \mathcal{M}, \mathcal{K}_{c}$ is totally disconnected and $\Phi_{c}$ is not defined in all of its complement, but it is well-defined at the critical value.) Parameter rays $\mathcal{R}_{M}(\theta)$ are defined as preimages of straight rays under $\Phi_{M}$. Their landing properties are obtained e.g. in [16]: each rational ray $\mathcal{R}_{M}(\theta)$ is landing at a point $c=\gamma_{M}(\theta) \in \partial \mathcal{M}$. When $\theta$ is preperiodic, then the critical value $c$ of $f_{c}$ is preperiodic, and the parameter $c$ is called a Misiurewicz point. The critical value $c \in \mathcal{K}_{c}$ has the same external angles as the parameter $c \in \mathcal{M}$. When $\theta$ is periodic, then $c$ is the root of a hyperbolic component (see below). Both in the dynamic plane of $f_{c}$ and in the parameter plane, the landing points of two or more rational rays are called pinching points. They are used to disconnect these sets into well-defined components, which are described combinatorially by rational numbers. Their structure is obtained from the dynamics, and transfered to the parameter plane. Pinching points with more than two branches are branch points.

Hyperbolic components of $\mathcal{M}$ consist of parameters, such that the corresponding polynomial has an attracting cycle. The root is the parameter on the boundary, such that the cycle has multiplier 1 . The boundary of a hyperbolic component contains a dense set of roots of satellite components. Each hyperbolic component has a unique center, where the corresponding cycle is superattracting. Centers or roots are dense at/in $\partial \mathcal{M}$. 


\subsection{Quasi-Conformal Mappings}

An orientation-preserving homeomorphism $\psi$ between domains in $\widehat{\mathbb{C}}$ is $K$-quasiconformal, $1 \leq K<\infty$, if it has two properties:

- It is weakly differentiable, so that its differential $d \psi=\partial \psi d z+\bar{\partial} \psi d \bar{z}$ is defined almost everywhere. This linear map is sending certain ellipses in the tangent space to circles. The Beltrami coefficient $\mu:=\bar{\partial} \psi / \partial \psi$ is defined almost everywhere. It encodes the direction and the dilatation ratio of the semi-axes for the ellipse field [5].

- The dilatation ratio is bounded globally by $K$, or $|\mu(z)| \leq(K-1) /(K+1)$ almost everywhere.

The chain rule for derivatives is satisfied for the composition of quasi-conformal mappings, and a 1-quasi-conformal mapping is conformal. Quasi-conformal mappings are absolutely continuous, Hölder continuous, and have nice properties regarding e.g. boundary behavior or normal families [10]. Given a measurable ellipse field (Beltrami coefficient) $\mu$ with $|\mu(z)| \leq m<1$ almost everywhere, the Beltrami differential equation $\bar{\partial} \psi=\mu \partial \psi$ on $\widehat{\mathbb{C}}$ has a unique solution with the normalization $\psi(z)=z+o(1)$ as $z \rightarrow \infty$. The dependence on parameters is described by the Ahlfors-Bers Theorem [5], which is behind some of our arguments, but will not be used explicitly here.

A $K$-quasi-regular mapping is locally $K$-quasi-conformal except for critical points, but it need not be injective globally. In Sect. 3.3, we will have a quasi-regular mapping $g$, such that all iterates are $K$-quasi-regular, and analytic in a neighborhood of $\infty$. Then a $g$-invariant field of infinitesimal ellipses is obtained as follows: it consists of circles in a neighborhood of $\infty$, i.e. $\mu(z)=0$ there, and it is pulled back with iterates of $g$. Now $\psi$ shall solve the corresponding Beltrami equation, i.e. send these ellipses to circles. By the chain rule, $f:=\psi \circ g \circ \psi^{-1}$ is mapping almost every infinitesimal circle to a circle, thus it is analytic.

\section{Quasi-Conformal Surgery}

As soon as the combinatorial assumptions on $g_{c}^{(1)}$ given here are satisfied, Thm. 1.1 yields a corresponding homeomorphism of $\mathcal{E}_{M}$. After formulating these general assumptions, the proof is sketched by constructing the quasi-quadratic mapping $g_{c}$ and the homeomorphism $h$. For some details, the reader will be referred to [8].

\subsection{Combinatorial Setting}

The following definitions may be illustrated by the example in Fig. 2. Further examples are mentioned in Sects. 4.2-4.4. When four parameter rays are landing 
in pairs at two pinching points of $\mathcal{M}$, this defines a strip in the parameter plane. Analogously, four dynamic rays define a strip in the dynamic plane. Our assumptions are formulated in terms of eight preperiodic angles

$$
0<\Theta_{1}^{-}<\Theta_{2}^{-}<\Theta_{3}^{-}<\Theta_{4}^{-}<\Theta_{4}^{+}<\Theta_{3}^{+}<\Theta_{2}^{+}<\Theta_{1}^{+}<1
$$

- The Misiurewicz points $a:=\gamma_{M}\left(\Theta_{1}^{-}\right)=\gamma_{M}\left(\Theta_{1}^{+}\right) \neq \gamma_{M}\left(\Theta_{4}^{-}\right)=\gamma_{M}\left(\Theta_{4}^{+}\right)=: b$ mark a compact, connected, full subset $\mathcal{E}_{M} \subset \mathcal{M}: \mathcal{E}_{M}=\mathcal{P}_{M} \cap \mathcal{M}$, where $\mathcal{P}_{M}$ is the closed strip bounded by the four parameter rays $\mathcal{R}_{M}\left(\Theta_{1}^{ \pm}\right), \mathcal{R}_{M}\left(\Theta_{4}^{ \pm}\right)$.

- For all $c \in \mathcal{E}_{M}$, the eight dynamic rays $\mathcal{R}_{c}\left(\Theta_{i}^{ \pm}\right)$shall be landing in pairs at four distinct points, i.e. $\gamma_{c}\left(\Theta_{i}^{-}\right)=\gamma_{c}\left(\Theta_{i}^{+}\right)$. (Equivalently, they are landing in this pattern for one $c_{0} \in \mathcal{E}_{M}$, and none of the eight angles is returning to $\left(\Theta_{1}^{-}, \Theta_{1}^{+}\right)$under doubling $\bmod 1$.) Four open strips are defined as follows, cf. Fig. 2: $V_{c}$ is bounded by $\mathcal{R}_{c}\left(\Theta_{1}^{ \pm}\right)$and $\mathcal{R}_{c}\left(\Theta_{2}^{ \pm}\right), W_{c}$ is bounded by $\mathcal{R}_{c}\left(\Theta_{2}^{ \pm}\right)$ and $\mathcal{R}_{c}\left(\Theta_{4}^{ \pm}\right), \widetilde{V}_{c}$ is bounded by $\mathcal{R}_{c}\left(\Theta_{1}^{ \pm}\right)$and $\mathcal{R}_{c}\left(\Theta_{3}^{ \pm}\right), \widetilde{W}_{c}$ is bounded by $\mathcal{R}_{c}\left(\Theta_{3}^{ \pm}\right)$ and $\mathcal{R}_{c}\left(\Theta_{4}^{ \pm}\right) . \mathcal{E}_{c} \subset \mathcal{K}_{c}$ is defined as the intersection of $\mathcal{K}_{c}$ with the closed strip $\overline{V_{c} \cup W_{c}}=\overline{\widetilde{V}_{c} \cup \widetilde{W}_{c}}$. Thus for parameters $c \in \mathcal{E}_{M}$, the critical value $c$ satisfies $c \in \mathcal{E}_{c}$.

- The first-return number $k_{v}$ is the smallest integer $k>0$, such that $f_{c}^{k}\left(V_{c}\right)$ meets (covers) $\mathcal{E}_{c}$. Equivalently, it is the largest integer $k>0$, such that $f_{c}^{k-1}$ is injective on $V_{c}$. Define $k_{w}, \widetilde{k}_{v}, \widetilde{k}_{w}$ analogously. They are independent of $c \in \mathcal{E}_{M}$. Now the main assumption on the dynamics, which makes finding the angles non-trivial, is that there is a (fixed) choice of signs with $f_{c}^{k_{v}-1}\left(V_{c}\right)=$ $\pm f_{c}^{\tilde{k}_{v}-1}\left(\widetilde{V}_{c}\right)$ and $f_{c}^{k_{w}-1}\left(W_{c}\right)= \pm f_{c}^{\widetilde{k}_{w}-1}\left(\widetilde{W}_{c}\right)$. The "orientation" is respected, i.e. with $z_{i}:=\gamma_{c}\left(\Theta_{i}^{ \pm}\right)$we have e.g. $f_{c}^{k_{v}-1}\left(z_{1}\right)= \pm f_{c}^{\widetilde{k}_{v}-1}\left(z_{1}\right)$ and $f_{c}^{k_{v}-1}\left(z_{2}\right)=$ $\pm f_{c}^{\widetilde{k}_{v}-1}\left(z_{3}\right)$.

If $a$ or $b$ is a branch point of $\mathcal{M}$, the last assumption implies that $\mathcal{E}_{M}$ is contained in a single branch, i.e. $\mathcal{E}_{M} \backslash\{a, b\}$ is a connected component of $\mathcal{M} \backslash\{a, b\}$.

\section{Definition 3.1 (Preliminary Mapping $g_{c}^{(1)}$ )}

Under these assumptions, with the unique choices of signs in the two strips, define $\eta_{c}:=f_{c}^{-\left(\widetilde{k}_{v}-1\right)} \circ\left( \pm f_{c}^{k_{v}-1}\right): V_{c} \rightarrow \widetilde{V}_{c}, \eta_{c}:=f_{c}^{-\left(\widetilde{k}_{w}-1\right)} \circ\left( \pm f_{c}^{k_{w}-1}\right): W_{c} \rightarrow \widetilde{W}_{c}$, and $\eta_{c}:=\mathrm{id}$ on $\mathbb{C} \backslash \overline{V_{c} \cup W_{c}}$ for $c \in \mathcal{E}_{M}$. Then define $g_{c}^{(1)}:=f_{c} \circ \eta_{c}$ and $\widetilde{g}_{c}^{(1)}:=f_{c} \circ \eta_{c}^{-1}$.

The three mappings are holomorphic and defined piecewise, thus they cannot be extended continuously. Each has "shift discontinuities" on six dynamic rays: e.g., consider $z_{0} \in \mathcal{R}_{c}\left(\Theta_{2}^{-}\right),\left(z_{n}^{\prime}\right) \subset V_{c}$ and $\left(z_{n}^{\prime \prime}\right) \subset W_{c}$ with $z_{n}^{\prime} \rightarrow z_{0}$ and $z_{n}^{\prime \prime} \rightarrow z_{0}$, then $\lim g_{c}^{(1)}\left(z_{n}^{\prime}\right)$ and $\lim g_{c}^{(1)}\left(z_{n}^{\prime \prime}\right)$ both exist and belong to $\mathcal{R}_{c}\left(\Theta_{3}^{-}\right)$, but they are shifted relative to each other along this ray. Neglecting these rays, $g_{c}^{(1)}$ and $\widetilde{g}_{c}^{(1)}$ are proper of degree 2. In the following section, $g_{c}^{(1)}$ will be replaced with a smooth mapping $g_{c}$, which is used to construct the homeomorphism $h$. Analogously, $\widetilde{g}_{c}^{(1)}$ yields $\widetilde{h}=h^{-1}$. 


\subsection{Construction of the Quasi-Quadratic Mapping $g_{c}$}

For $c \in \mathcal{E}_{M}$, we construct a quasi-regular mapping $g_{c}$ coinciding with $g_{c}^{(1)}$ on $\mathcal{K}_{c}$. By employing the Boettcher conjugation $\Phi_{c}$, the work will be done in the exterior of the unit disk $\overline{\mathbb{D}}$. This is convenient when $c \in \mathcal{E}_{M}$, and essential to construct the homeomorphism $h$ in the exterior. In $\widehat{\mathbb{C}} \backslash \mathcal{K}_{c}$ we have $g_{c}^{(1)}=\Phi_{c}^{-1} \circ G^{(1)} \circ \Phi_{c}$, where $G^{(1)}: \widehat{\mathbb{C}} \backslash \overline{\mathbb{D}} \rightarrow \widehat{\mathbb{C}} \backslash \overline{\mathbb{D}}$ is discontinuous on six straight rays, and given by compositions of $F(z):=z^{2}$ in the regions between these rays - it is independent of $c$ in particular.

1. First construct smooth domains $U, U^{\prime}$ with $\overline{\mathbb{D}} \subset U$ and $\bar{U} \subset U^{\prime}$, and a smooth mapping $G: U \backslash \overline{\mathbb{D}} \rightarrow U^{\prime} \backslash \overline{\mathbb{D}}$. It shall be proper of degree 2 and coincide with $G^{(1)}$ except in sectors around those six rays $\mathcal{R}\left(\Theta_{i}^{ \pm}\right)$, where $G^{(1)}$ has a shift discontinuity. The sectors are of the form $\left|\arg z-2 \pi \Theta_{i}^{ \pm}\right|<s \log |z|$, and there $G$ is chosen conveniently as a 1-homogeneous function of $\log z-\mathrm{i} 2 \pi \Theta_{i}^{ \pm}$, thus ensuring that the dilatation bound does not explode at the vertex of the sector. (This simplifies the construction of [3], which employed a pullback of quadrilaterals.) The domains are chosen in a finite recursion, employing that some iterate of $G^{(1)}$ is strictly expanding [8, Sect. 5.2]. Since any orbit is visiting at most two of the sectors, the dilatation of all iterates of $G$ is bounded uniformly.

2. Choose the radius $R>1$ and the conformal mapping $H: \widehat{\mathbb{C}} \backslash \overline{U^{\prime}} \rightarrow \widehat{\mathbb{C}} \backslash \overline{\mathbb{D}}_{R^{2}}$ with the normalization $H(z)=z+\mathcal{O}(1 / z)$ at $\infty$ (which determines $R$ and $H$ uniquely). Extend $H$ to a quasi-conformal mapping $H: \widehat{\mathbb{C}} \backslash U \rightarrow \widehat{\mathbb{C}} \backslash \mathbb{D}_{R}$ with $F \circ H=H \circ G$ on $\partial U$. Define the extended $G: \widehat{\mathbb{C}} \backslash \overline{\mathbb{D}} \rightarrow \widehat{\mathbb{C}} \backslash \overline{\mathbb{D}}$ by $G:=H^{-1} \circ F \circ H$ on $\widehat{\mathbb{C}} \backslash U$. Now $G$ is proper of degree 2, quasi-regular, and the dilatation of $G^{n}$ is bounded by some $K$ uniformly in $n$. Finally, extend $H$ to a mapping $H: \widehat{\mathbb{C}} \backslash \overline{\mathbb{D}} \rightarrow \widehat{\mathbb{C}} \backslash \overline{\mathbb{D}}$ by recursive pullbacks, such that $F \circ H=H \circ G$ everywhere, then $H$ is $K$-quasi-conformal. Cf. Fig. 4.

3. Now, set $g_{c}:=g_{c}^{(1)}$ on $\mathcal{K}_{c}$ and $g_{c}:=\Phi_{c}^{-1} \circ G \circ \Phi_{c}$ on $\widehat{\mathbb{C}} \backslash \mathcal{K}_{c}$. Then $g_{c}$ is a quasi-quadratic mapping, i.e. proper of degree 2 , with a uniform bound on the dilatation of the iterates, with $\bar{\partial} g_{c}=0$ a.e. on $\mathcal{K}_{c}$, and analytic in a neighborhood of $\infty$ with $g_{c}(z)=z^{2}+\mathcal{O}(1)$. (It is continuous at $\gamma_{c}\left(\Theta_{i}^{ \pm}\right)$by Lindelöf's Theorem.)

Now suppose that $c \in \mathcal{P}_{M} \backslash \mathcal{E}_{M}$ with $\Phi_{M}(c) \in U^{\prime}$. Then $\mathcal{K}_{c}$ is totally disconnected, and $\Phi_{c}$ is not defined in all of $\widehat{\mathbb{C}} \backslash \mathcal{K}_{c}$. It can be defined, however, in a domain mapped to the six sectors and to $\widehat{\mathbb{C}} \backslash \bar{U}$ by $\Phi_{c}[3,8]$. Thus $g_{c}$ is defined in this case as well, by matching $g_{c}^{(1)}$ with $\Phi_{c}^{-1} \circ G \circ \Phi_{c}$.

In the following section, we shall construct an invariant ellipse field for the quasiquadratic $g_{c}$, and employ it to straighten $g_{c}$, i.e. to conjugate it to a quadratic polynomial $f_{d}$. Then we set $h(c):=d$. If we had skipped step $2, g_{c}$ would not be a quasi-quadratic mapping $\mathbb{C} \rightarrow \mathbb{C}$, but a quasi-regular quadratic-like mapping 


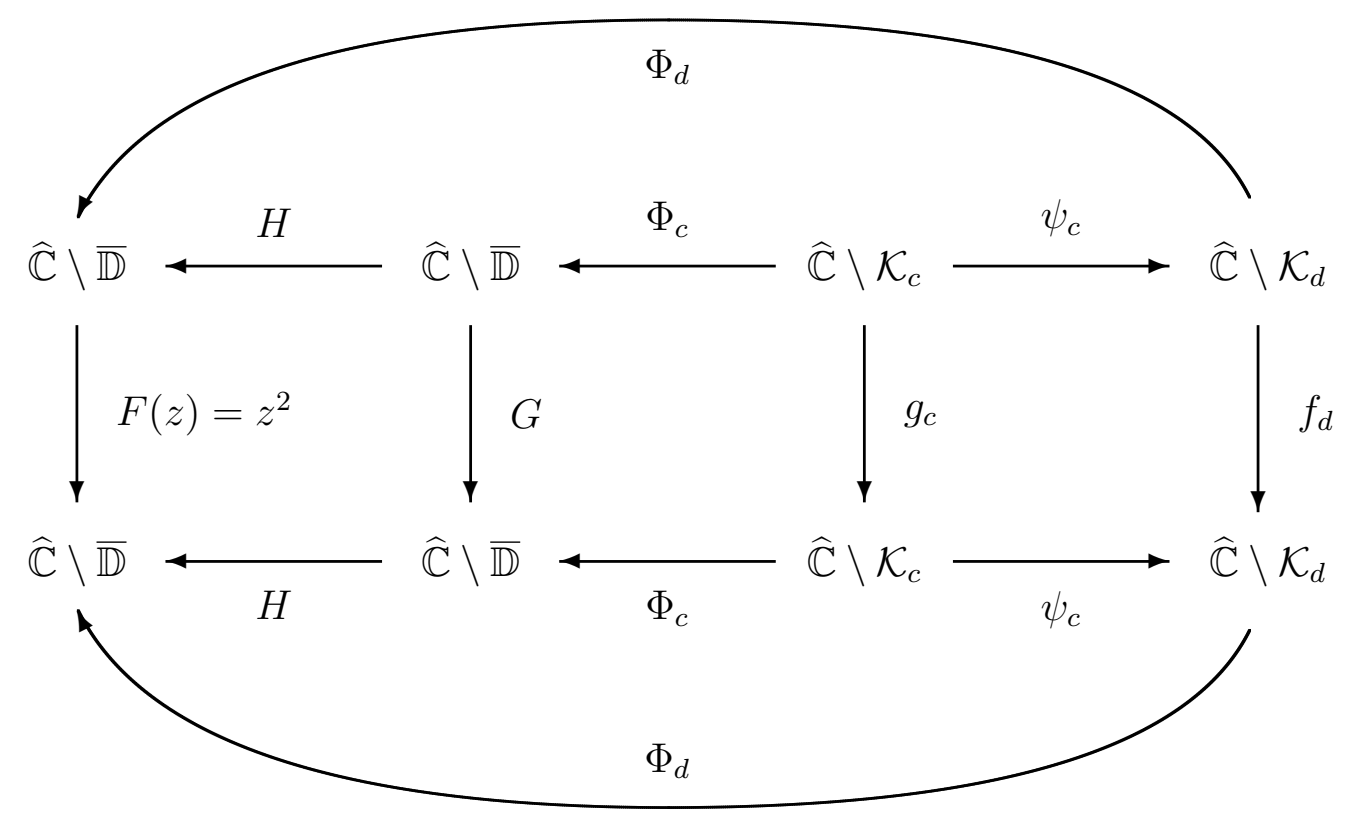

Figure 4: Construction and straightening of $g_{c}$ by employing mappings in the exterior of the unit disk. If the filled Julia sets are not connected, the diagram is well-defined and commuting on smaller neighborhoods of $\infty$.

(cf. $[6,8]$ ) between bounded domains $U_{c} \rightarrow U_{c}^{\prime}$. This distinction is related to possible alternative techniques:

\section{Remark 3.2 (Alternative Techniques)}

1. The classical techniques would be as follows $[1,2]$ : after the quasi-regular quadratic-like mapping $g_{c}: U_{c} \rightarrow U_{c}^{\prime}$ is constructed, it is not extended to $\mathbb{C}$, but it is first conjugated to an analytic quadratic-like mapping, employing an invariant ellipse field in $U_{c}^{\prime}$. Then the latter mapping is straightened to a polynomial by the Straightening Theorem [6]. With this approach, it will not be possible to extend the homeomorphism $h$ to the exterior of $\mathcal{M}$.

2. Here we shall use the same techniques as in [3]: having extended $g_{c}$ to $\widehat{\mathbb{C}}$, it will be easy to straighten. Instead of applying the Straightening Theorem, its proof [5] was adapted into the construction of $g_{c}$. This approach makes the extension of $h$ to the exterior of $\mathcal{M}$ possible. By applying this technique to the construction of $g_{c}$ and $h(c)$ for $c \in \mathcal{E}_{M}$ as well, the proofs of bijectivity, continuity, and of landing properties (Sect. 4.1) are simplified.

3. Alternatively, $g_{c}: U_{c} \rightarrow U_{c}^{\prime}$ could be constructed as a quasi-regular quadraticlike mapping on a bounded domain, and be straightened without extending it to $\widehat{\mathbb{C}}$ first, by incorporating the alternative proofs of the Straightening Theorem according to [6]. This proof is more involved, but it has the advantage that the mapping $H$ can be chosen more freely on $\overline{U^{\prime}} \backslash U$, e.g. such that it is the identity on $\mathcal{R}\left(\Theta_{1}^{ \pm}\right)$ and $\mathcal{R}\left(\Theta_{4}^{ \pm}\right)$[8]. Then $h$ would be the identity on the corresponding parameter rays, which makes it easier to paste different homeomorphisms together. 


\section{3 $h$ is a Homeomorphism}

For $c \in \mathcal{E}_{M}$, or $c \in \mathcal{P}_{M} \backslash \mathcal{E}_{M}$ with $\Phi_{M}(c) \in U^{\prime}$, the quasi-quadratic mapping $g_{c}$ was constructed in the previous section. Now construct the $g_{c}$-invariant ellipse field $\mu$ by pullbacks with $g_{c}$, such that $\mu=0$ in a neighborhood of $\infty$ and a.e. on $\mathcal{K}_{c}$. It is bounded by $(K-1) /(K+1)$, since the dilatation of all iterates $g_{c}^{n}$ is bounded by $K$. Denote by $\psi_{c}$ the solution of the Beltrami equation $\bar{\partial} \psi=\mu \partial \psi$, normalized by $\psi_{c}(z)=z+\mathcal{O}(1 / z)$, which is mapping the infinitesimal ellipses described by $\mu$ to circles. Now $\psi_{c} \circ g_{c} \circ \psi_{c}^{-1}$ is analytic on $\widehat{\mathbb{C}}$ and proper of degree 2 , thus a quadratic polynomial of the form $f_{d}(z)=z^{2}+d$. In a neighborhood of $\infty, H \circ \Phi_{c} \circ \psi_{c}^{-1}$ is conjugating $f_{d}$ to $F$, cf. Fig. 4. By the uniqueness of the Boettcher conjugation, this mapping equals $\Phi_{d}$. Recursive pullbacks show equality in $\widehat{\mathbb{C}} \backslash \mathcal{K}_{d}$, if $\mathcal{K}_{c}$ and $\mathcal{K}_{d}$ are connected, i.e. for $c \in \mathcal{E}_{M}$. Otherwise, equality holds on an $f_{d}$-forward-invariant domain of $\Phi_{d}$, which may be chosen to include the critical value $d$.

We set $h(c):=d=\psi_{c}(c)$. If $c \in \mathcal{E}_{M}$, a combinatorial argument shows $d \in \mathcal{E}_{M}$. If $c \in \mathcal{P}_{M} \backslash \mathcal{E}_{M}$ with $\Phi_{M}(c) \in U^{\prime}$, we have

$$
\Phi_{M}(d)=\Phi_{d}(d)=\Phi_{d} \circ \psi_{c}(c)=H \circ \Phi_{c}(c)=H \circ \Phi_{M}(c) .
$$

Denote by $\widetilde{\mathcal{P}}_{M}$ the closed strip that is bounded by the four curves $\Phi_{M}^{-1} \circ H\left(\mathcal{R}\left(\Theta_{i}^{ \pm}\right)\right)$, $i=1,4$, which are quasi-arcs. Now $h$ is extended to $h: \mathcal{P}_{M} \rightarrow \widetilde{\mathcal{P}}_{M}$ by setting

$$
h:=\Phi_{M}^{-1} \circ H \circ \Phi_{M}: \mathcal{P}_{M} \backslash \mathcal{E}_{M} \rightarrow \widetilde{\mathcal{P}}_{M} \backslash \mathcal{E}_{M} .
$$

By (2), this agrees with the definition of $h(c)$ by straightening $g_{c}$, if $\Phi_{M}(c) \in U^{\prime}$. Now (3) shows that $h$ is bijective and $K$-quasi-conformal in the exterior of $\mathcal{E}_{M}$. We will see that $h$ is bijective and continuous on $\mathcal{E}_{M}$. Let us remark that for $c \in \mathcal{E}_{M}$, the value of $d=h(c)$ does not depend on the choices made in the construction of $G$ and $H$, since $\psi_{c}$ is a hybrid-equivalence [6]. The proof of bijectivity in [2] relied on this independence, but the following one is simplified by employing $H$ :

For $d \in \mathcal{E}_{M}$, consider $\widetilde{g}_{d}^{(1)}$ according to Def. 3.1, and define the quasi-quadratic mapping $\widetilde{g}_{d}$ with $\widetilde{g}_{d}:=\widetilde{g}_{d}^{(1)}$ on $\mathcal{K}_{d}$ and $\widetilde{g}_{d}:=\Phi_{d}^{-1} \circ \widetilde{G} \circ \Phi_{d}$ in $\widehat{\mathbb{C}} \backslash \mathcal{K}_{d}$, where $\widetilde{G}:=$ $H \circ F \circ H^{-1}$. To see that this choice is possible, note that $H$ is mapping the region $V \subset U \backslash \overline{\mathbb{D}}$ (corresponding to $V_{c}$ ) to a distorted version of $\tilde{V}$. There we have $\widetilde{G}^{(1)}=F^{2-k_{v}} \circ\left( \pm F^{\widetilde{k}_{v}-1}\right)$. Observing that $F=H \circ G \circ H^{-1}$ and $H$ commutes with \pm id on the set in question, we have $\widetilde{G}^{(1)}=H \circ G^{2-k_{v}} \circ\left( \pm G^{\bar{k}_{v}-1}\right) \circ H^{-1}$. Following the orbit and applying the piecewise definition of $G^{(1)}$ yields $\widetilde{G}^{(1)}=H \circ F \circ H^{-1}$. Together with the same result in other regions, this justifies the definition of $\widetilde{G}$, i.e. $\widetilde{g}_{d}$ is quasi-quadratic. Now $\widetilde{h}(d)$ is defined by straightening $\widetilde{g}_{d}$. - Suppose that $c \in \mathcal{E}_{M}$ and $d=h(c)$, then $f_{d}=\psi_{c} \circ g_{c} \circ \psi_{c}^{-1}$ and by its definition in terms of $H=\Phi_{d} \circ \psi_{c} \circ \Phi_{c}^{-1}$, we have $\widetilde{g}_{d}=\psi_{c} \circ f_{c} \circ \psi_{c}^{-1}$. Therefore $c=\widetilde{h}(d)$ and $\widetilde{\psi}_{d}=\psi_{c}^{-1}$. $\widetilde{h} \circ h=\mathrm{id}$ and the converse result imply that $h: \mathcal{E}_{M} \rightarrow \mathcal{E}_{M}$ is bijective with $h^{-1}=\widetilde{h}$.

By (3), $h$ is quasi-conformal in the exterior of $\mathcal{E}_{M}$. The interior of $\mathcal{E}_{M}$ consists of a countable family of hyperbolic components, plus possibly a countable family of 
non-hyperbolic components. The former are parametrized by multiplier maps, the latter by transforming invariant line fields. In both cases, $h$ is given by a composition of these analytic parametrizations $[2,8]$. It remains to show that $h$ is continuous at $c_{0} \in \partial \mathcal{E}_{M}$ : suppose $c_{n} \rightarrow c_{0}, d_{n}=h\left(c_{n}\right), d_{0}=h\left(c_{0}\right)$. By bijectivity we have $d_{0} \in \partial \mathcal{E}_{M}=\mathcal{E}_{M} \cap \partial \mathcal{M}$. It does not matter if $c_{n}$ belongs to $\mathcal{E}_{M}$ or not. (Now we employ the definition of $h$ by straightening $g_{c}$, which is equivalent to (3). One special case requires extra treatment: when some $\gamma_{c}\left(\Theta_{i}^{ \pm}\right)$is iterated to $\gamma_{c}\left(\Theta_{1}^{ \pm}\right)$, and $c_{0}=\gamma_{M}\left(\Theta_{i}^{ \pm}\right)$, then $g_{c_{0}}$ is not defined.) It is sufficient to show $d_{n} \rightarrow d_{*} \Rightarrow d_{*}=d_{0}$. Since the $K$ quasi-conformal mappings $\psi_{n}$ are normalized, there is a $K$-quasi-conformal $\Psi$ and a subsequence $\psi_{c_{n}^{\prime}} \rightarrow \Psi$, uniformly on $\widehat{\mathbb{C}}[10]$. We have $\psi_{c_{n}^{\prime}} \circ g_{c_{n}^{\prime}} \circ \psi_{c_{n}^{\prime}}^{-1} \rightarrow \Psi \circ g_{c_{0}} \circ \Psi^{-1}$ and $\psi_{c_{n}} \circ g_{c_{n}} \circ \psi_{c_{n}}^{-1}=f_{d_{n}} \rightarrow f_{d_{*}}$, thus $\Psi \circ \psi_{c_{0}}^{-1}$ is a quasi-conformal conjugation from $f_{d_{0}}$ to $f_{d_{*}}$. Although it need not be a hybrid-equivalence, $d_{0} \in \partial \mathcal{M}$ implies $d_{*}=d_{0}$ [6]. By the same arguments, or by the Closed Graph Theorem, $h^{-1}$ is continuous as well. Thus $h: \mathcal{P}_{M} \rightarrow \widetilde{\mathcal{P}}_{M}$ is a homeomorphism mapping $\mathcal{E}_{M} \rightarrow \mathcal{E}_{M}$.

\subsection{Further Properties of $h$}

Since $h$ is analytic in the interior of $\mathcal{E}_{M}$ and quasi-conformal in the exterior, it is natural to ask if it is quasi-conformal everywhere. Branner and Lyubich are working on a proof employing quasi-regular quadratic-like germs. Maybe an alternative proof can be given by constructing a homotopy from $f_{c}$ to $\widetilde{g}_{d}$, thus from id to $h$.

The dynamics of $h$ on $\mathcal{E}_{M}$ is simple: set $c_{0}:=\gamma_{M}\left(\Theta_{2}^{ \pm}\right)$and $c_{n}:=h^{n}\left(c_{0}\right), n \in \mathbb{Z}$. The connected component of $\mathcal{E}_{M}$ between the two pinching points $c_{n}$ and $c_{n+1}$ is a fundamental domain for $h^{ \pm 1}$. These are accumulating at the Misiurewicz points $a$ and $b$, and the method of [17] yields a linear scaling behavior. Thus $h$ and $h^{-1}$ are Lipschitz continuous at $a$ and $b$ (and Hölder continuous at all Misiurewicz points). For $c \in \mathcal{E}_{M} \backslash\{a, b\}$ we have $h^{n}(c) \rightarrow b$ as $n \rightarrow \infty$ and $h^{n}(c) \rightarrow a$ as $n \rightarrow-\infty$.

\section{Related Results and Possible Generalizations}

Further results and examples from [8] are sketched, and we present some ideas on surgery for general one-parameter families.

\subsection{Combinatorial Surgery}

The unit circle $\partial \mathbb{D}$ is identified with $S^{1}:=\mathbb{R} / \mathbb{Z}$ by the parametrization $\exp (\mathrm{i} 2 \pi \theta)$. For $h$ constructed from $g_{c}^{(1)}$ according to Thm. 1.1, recall the mappings $F, G, H$ : $\widehat{\mathbb{C}} \backslash \overline{\mathbb{D}} \rightarrow \widehat{\mathbb{C}} \backslash \overline{\mathbb{D}}$ from Sect. 3.2. Denote their boundary values by $\mathbf{F}, \mathbf{G}, \mathbf{H}: S^{1} \rightarrow S^{1}$. Thus $\mathbf{F}(\theta)=2 \theta \bmod 1$ and $\mathbf{G}$ is piecewise linear. Now $\mathbf{H}$ is the unique orientationpreserving circle homeomorphism conjugating $\mathbf{G}$ to $\mathbf{F}, \mathbf{H} \circ \mathbf{G} \circ \mathbf{H}^{-1}=\mathbf{F} . \mathbf{H}(\theta)$ is computed numerically from the orbit of $\theta$ under $\mathbf{G}$ as follows: for $n \in \mathbb{N}$, the 
$n$-th binary digit of $\mathbf{H}(\theta)$ is 0 if $0 \leq \mathbf{G}^{n-1}(\theta)<1 / 2$, and 1 if $1 / 2 \leq \mathbf{G}^{n-1}(\theta)<1$. For rational angles, the (pre-) periodic sequence of digits is obtained from a finite algorithm.

In the exterior of $\mathcal{E}_{M}, h$ is represented by $H$ according to (3). Applying this formula to parameter rays and employing Lindelöf's Theorem shows: $\mathcal{R}_{M}(\theta)$ is landing at $c \in \partial \mathcal{E}_{M}$, iff $\mathcal{R}_{M}(\mathbf{H}(\theta))$ is landing at $h(c)$. If $c$ is a Misiurewicz point or a root, then $\theta$ is rational, and $\mathbf{H}(\theta)$ is computed exactly. In this sense, $d=h(c)$ is determined combinatorially. Alternatively, one can construct the critical orbit of $g_{c}^{(1)}$ and the Hubbard tree of $f_{d}$. The simplest case is given when the critical orbit meets $\mathcal{E}_{c}$ only once: then the orbit of $c$ under $g_{c}^{(1)}$ is the same as the orbit of $\eta_{c}(c)$ under $f_{c}$.

Regularity properties of $\mathbf{H}$ are discussed in [8, Sect. 9.2]. $\mathbf{H}$ has Lipschitz or Hölder scaling properties at all rational angles. $\mathbf{H}$ and $\mathbf{H}^{-1}$ are Hölder continuous with the optimal exponents $\widetilde{k}_{v} / k_{v}$ and $k_{w} / \widetilde{k}_{w}$. Since $H$ is $K$-quasi-conformal, Mori's Theorem [10] says that $\mathbf{H}^{ \pm 1}$ is $1 / K$-Hölder continuous. Thus we have the lower bound $K \geq \max \left(k_{v} / \widetilde{k}_{v}, \widetilde{k}_{w} / k_{w}\right)$, independent of the choices made in the construction of $h: \mathcal{P}_{M} \backslash \mathcal{E}_{M} \rightarrow \widetilde{\mathcal{P}}_{M} \backslash \mathcal{E}_{M}$. By a piecewise construction we obtain a homeomorphism $h: \mathcal{M} \rightarrow \mathcal{M}$, which extends to a homeomorphism of $\mathbb{C}$, but such that no extension can be quasi-conformal.

\subsection{Homeomorphisms at Misiurewicz Points}

A homeomorphism $h: \mathcal{E}_{M} \rightarrow \mathcal{E}_{M}$ according to Thm. 1.1 is expanding at the Misiurewicz point $a$. Asymptotically, $\mathcal{M}$ shows a linear scaling behavior at $a$. (In Fig. 3, you can observe the asymptotic self-similarity of $\mathcal{M}$ at $a$, and similarity between $\mathcal{M}$ at $c \approx c_{n}$ and $\mathcal{K}_{c_{n}}$ at $z \approx 0$.) Now $h$ is asymptotically linear in a "macroscopic" sense, e.g. there is an asymptotically linear sequence of fundamental domains, but this is not true pointwise. These results are obtained by combining the techniques from [17] with the combinatorial description of $h$ according to Sect. 4.1: consider a suitable sequence $c_{n} \rightarrow a$. If the critical orbit of $f_{c_{n}}$ travels through $\mathcal{E}_{c_{n}}$ once, then $h$ is asymptotically linear on the sequence, but it is not if the orbit meets $\mathcal{E}_{c_{n}}$ twice. Conversely, given a branch at some Misiurewicz point $a$, is there an appropriate homeomorphism $h$ ? We only need to find a combinatorial construction of $g_{c}^{(1)}$. This is done in [8] e.g. for all $\beta$-type Misiurewicz points. (Here $\mathcal{P}_{M}$ and $\overline{V_{c} \cup W_{c}}$ are sectors, not strips.) The author's research was motivated by discussions with D. Schleicher, who had worked on the construction of dynamics in the parameter plane before.

\subsection{Edges, Frames, and Piecewise Constructions}

For parameters $c$ in the $p / q$-limb of $\mathcal{M}$, the filled Julia set $\mathcal{K}_{c}$ has $q$ branches at the fixed point $\alpha_{c}$ of $f_{c}$. A connected subset $\mathcal{E}_{c} \subset \mathcal{K}_{c}$ is a dynamic edge of order $n$, if $f_{c}^{n-1}$ is injective on $\mathcal{E}_{c}$ and $f_{c}^{n-1}\left(\mathcal{E}_{c}\right)$ is the part of $\mathcal{K}_{c}$ between $\alpha_{c}$ and $-\alpha_{c}$. (More precisely, $f_{c}^{n-1}$ shall be injective in a neighborhood of the edge without its vertices.) 
The edge is characterized by the external angles at the vertices. As $c$ varies, it may still be defined, or it may cease to exist after a bifurcation of preimages of $\alpha_{c}$. Now $\mathcal{E}_{M} \subset \mathcal{M}_{p / q}$ is a parameter edge, if for all $c \in \mathcal{E}_{M}$ the dynamic edge $\mathcal{E}_{c}$ (with given angles) exists and satisfies $c \in \mathcal{E}_{c}$, and if $\mathcal{E}_{M}$ has the same external angles as $\mathcal{E}_{c}$. In Figs. $2-3, \mathcal{E}_{M}$ is the parameter edge of order 4 in $\mathcal{M}_{1 / 3}$.

$\mathcal{M}_{p / q}$ contains a little Mandelbrot set $\mathcal{M}^{\prime}=c_{0} * \mathcal{M}$ of period $q$ (cf. Sect. 4.4). If a parameter edge $\mathcal{E}_{M}$ is behind $c_{0} *(-1)$, there is a homeomorphism $h: \mathcal{E}_{M} \rightarrow \mathcal{E}_{M}$ analogous to that of Figs. 2-3. Behind the $\alpha$-type Misiurewicz point $c_{0} *(-2)$, edges can be decomposed into subedges and frames [8, Sect. 7]. These frames are constructed recursively, like the intervals in the complement of the middle-third Cantor set. A family of homeomorphisms on subedges shows that all frames on the same edge are mutually homeomorphic, and they form a finer decomposition than the fundamental domains of a single homeomorphism. By permuting the frames (in a monotonous way), new homeomorphisms $h$ are defined piecewise. These may have properties that are not possible when $h$ is constructed from a single surgery. E.g., in contradiction to Sect. 3.4, $h$ can be constructed such that it is not Lipschitz continuous or not even Hölder continuous at the vertex $a$ of $\mathcal{E}_{M}$. Or it can map a Misiurewicz point with two external angles to a parameter with irrational angles, which is not a Misiurewicz point.

The notions of edges and frames can be generalized: for parameters $c$ behind the root of a hyperbolic component, the filled Julia set $\mathcal{K}_{c}$ contains two corresponding pre-characteristic points, which take the roles of $\pm \alpha_{c}$.

\subsection{Tuning and Composition of Homeomorphisms}

For a center $c_{0}$ of period $p$, there is a "little Mandelbrot set" $\mathcal{M}^{\prime} \subset \mathcal{M}$ and a tuning map $\mathcal{M} \rightarrow \mathcal{M}^{\prime}, x \mapsto y=c_{0} * x$ with $0 \mapsto c_{0}$. Now $\mathcal{K}_{y}$ contains a "little Julia set" $\mathcal{K}_{y, p}$ around 0 , where $f_{y}^{p}$ is conjugate to $f_{x}$ on $\mathcal{K}_{x}[6,7]$. A homeomorphism $h: \mathcal{E}_{M} \rightarrow \mathcal{E}_{M}$ according to Thm. 1.1 is compatible with tuning in two different ways:

- If $c_{0} \in \mathcal{E}_{M}$, then $h$ is mapping $\mathcal{M}^{\prime}$ to the little Mandelbrot set at $h\left(c_{0}\right)$ : $h\left(c_{0} * x\right)=\left(h\left(c_{0}\right)\right) * x$. Cf. [2].

- For any center $c_{0} \in \mathcal{M}$, set $\mathcal{E}_{M}^{\prime}:=c_{0} * \mathcal{E}_{M} \subset \mathcal{M}$. A new homeomorphism $h^{\prime}: \mathcal{E}_{M}^{\prime} \rightarrow \mathcal{E}_{M}^{\prime}$ is obtained by composition, i.e. $h^{\prime}\left(c_{0} * x\right):=c_{0} *(h(x))$. Now $\mathcal{E}_{M}^{\prime}$ is obtained by disconnecting $\mathcal{M}$ at a countable family of pinching points, but $h^{\prime}$ has a natural extension to all of these "decorations" (except for two): the mapping $\eta_{c}$ that produced the homeomorphism $h$ is transferred by cutting the little Julia set into strips. The required pinching points do not bifurcate when the parameter $y$ is in a decoration of $\mathcal{E}_{M}^{\prime}$, thus the new piecewise construction $\eta_{y}^{\prime}$ works in a whole strip. An example is shown in Fig. 5 (left). 
The same principle applies, e.g., to crossed renormalization [12], or to the BrannerDouady homeomorphism $\Phi_{A}: \mathcal{M}_{1 / 2} \rightarrow \mathcal{T} \subset \mathcal{M}_{1 / 3}$ : suppose that $\mathcal{E}_{M} \subset \mathcal{M}_{1 / 2}$ and $h: \mathcal{E}_{M} \rightarrow \mathcal{E}_{M}$ is constructed according to Thm. 1.1, i.e. from a combinatorial $g_{c}^{(1)}$ according to Def. 3.1. Then $\mathcal{E}_{M}^{\prime}:=\Phi_{A}\left(\mathcal{E}_{M}\right)$ is a subset of $\mathcal{M}_{1 / 3}$, where a countable family of decorations was cut off. Again $h^{\prime}:=\Phi_{A} \circ h \circ \Phi_{A}^{-1}: \mathcal{E}_{M}^{\prime} \rightarrow \mathcal{E}_{M}^{\prime}$ extends to a whole strip by transferring the combinatorial construction of $g_{c}^{(1)}$. If, e.g., $h$ is a suitable homeomorphism on the edge from $\gamma_{M}(5 / 12)$ to $\gamma_{M}(11 / 24)$, then $h^{\prime}$ is the homeomorphism of Figs. 2, 3.
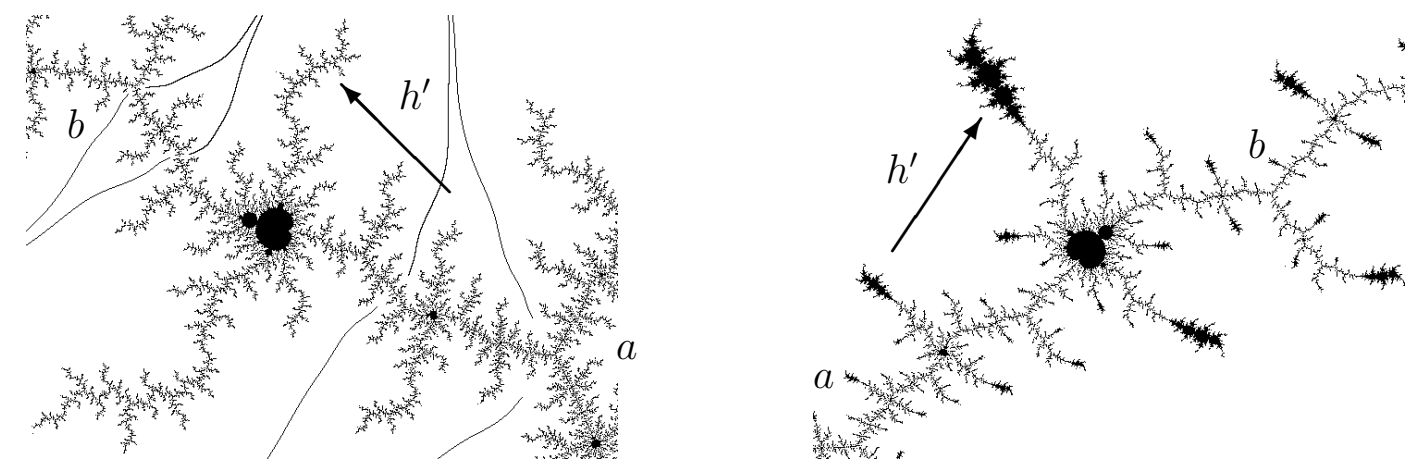

Figure 5: Two homeomorphisms $h^{\prime}: \mathcal{E}_{M}^{\prime} \rightarrow \mathcal{E}_{M}^{\prime}$ obtained from a similar construction as $h: \mathcal{E}_{M} \rightarrow \mathcal{E}_{M}$ in Figs. 2-3. Left: tuning with the center $c_{0}=-1$ yields an edge in the limb $\mathcal{M}_{1 / 2} \subset \mathcal{M}$. The eight angles $\Theta_{i}^{ \pm}$are obtained by tuning those of Fig. 2, i.e. replacing the digits 0 by 01 and 1 by $10 . h^{\prime}$ is defined not only on $c_{0} * \mathcal{E}_{M}$, but on a strip including all decorations. Right: part of the parameter space of cubic polynomials with a persistently indifferent fixed point. The connectedness locus contains copies of a quadratic Siegel Julia set [4]. Again, $h^{\prime}$ is defined in a strip containing a countable family of decorations attached to the copy of $\mathcal{E}_{M}$.

\subsection{Other Parameter Spaces}

In Thm. 1.1 we obtained homeomorphisms $h: \mathcal{E}_{M} \rightarrow \mathcal{E}_{M}$ of suitable subsets $\mathcal{E}_{M} \subset \mathcal{M}$, but the method is not limited to quadratic polynomials. To apply it to other onedimensional families of polynomials or rational mappings, these mappings must be characterized dynamically. The polynomials of degree $d$ form a $(d-1)$-dimensional family (modulo affine conjugation). Suppose that a one-dimensional subfamily $f_{c}$ is defined by one or more of the following critical relations:

- A critical point of $f_{c}$ is degenerate, or one critical point is iterated to another one, or critical orbits are related by $f_{c}$ being even or odd.

- A critical point is preperiodic or periodic (superattracting).

- There is a persistent cycle with multiplier $\rho, 0<|\rho| \leq 1$. This cycle is always "catching" one of the critical points, but the choice may change. 
An appropriate combination of such relations defines a one-parameter family $f_{c}$, where the coefficients and the critical points of $f_{c}$ are algebraic in $c$. Locally in the parameter space, there is one active or free critical point $\omega_{c}$, whose orbit determines the qualitative dynamics. The other critical points are either linked to $\omega_{c}$, or their behavior is independent of $c$. The connectedness locus $\mathcal{M}_{f}$ contains the parameters $c$, such that the filled Julia set $\mathcal{K}_{c}$ of $f_{c}$ is connected, equivalently $f_{c}^{n}\left(\omega_{c}\right) \nrightarrow \infty$, or $\omega_{c} \in \mathcal{K}_{c}$. In general $\omega_{c}$ is not defined globally by an analytic function of $c$, since it may be a multi-valued algebraic function of $c$, or since a persistent cycle may catch different critical points. But looking at specific families, it will be possible to define $\Phi_{M}$ and parameter rays for suitable subsets of the parameter space, and to understand their landing properties.

Then an analogue of Thm. 1.1 can be proved: for a piecewise defined $g_{c}^{(1)}$, a quasipolynomial mapping $g_{c}$ is constructed analogously to Sect. 3.2, and straightened to a polynomial. By the critical relations and by normalizing conditions, it will be of the form $f_{d}$, and we set $h(c):=d$. (At worst, the normalizing conditions will allow finitely many choices for $d$.) Note that this procedure will not work, if our family $f_{c}$ is an arbitrary submanifold of the $(d-1)$-dimensional family of all polynomials, and not defined by critical relations.

When such a theorem is proved, the remaining creative step is the combinatorial definition of $\mathcal{E}_{M}$ and $g_{c}^{(1)}$. Some examples can be obtained in the following way: when a non-degenerate critical point is active, the connectedness locus $\mathcal{M}_{f}$ will contain copies $\mathcal{M}^{\prime}$ of $\mathcal{M}$ [11]. Starting from a homeomorphism $h: \mathcal{E}_{M} \rightarrow \mathcal{E}_{M}, \mathcal{M}^{\prime}$ contains a decorated copy of $\mathcal{E}_{M}$, and the corresponding homeomorphism $h^{\prime}$ extends to all decorations by an appropriate definition of $g_{c}^{(1)}$ - the angles $\Theta_{i}^{ \pm}$are seen at the copy of a quadratic Julia set within $\mathcal{K}_{c}$, where some iterate of $f_{c}$ is conjugate to a quadratic polynomial. It remains to check that no other critical orbit is passing through $\overline{V_{c} \cup W_{c}}$, then $g_{c}^{(1)}$ is well-defined. An example is given in Fig. 5 (right).

The rational mappings of degree $d$ form a $(2 d-2)$-dimensional family (modulo Möbius conjugation). Suppose that a one-dimensional subfamily $f_{c}$ is defined by critical relations. When there are one or more persistently (super-) attracting cycles, then $\mathcal{K}_{c}$ shall be the complement of the basin of attraction, and $\mathcal{M}_{f}$ shall contain those parameters, such that the local free critical point is not attracted. $\partial \mathcal{M}_{f}$ will be the bifurcation locus [11]. If the persistent cycles are superattracting, we can define dynamic rays and parameter rays by the Boettcher conjugation. When the topology and the landing properties are understood sufficiently well, homeomorphisms can be constructed by quasi-conformal surgery.

An example is provided by cubic Newton methods, cf. $[6,18,14]$ : $f_{c}$ has three superattracting fixed points and one free critical point. Parts of the parameter space are shown in Fig. 6. By dynamic rays in the adjacent immediate basins of two fixed points, the Julia set is cut into "strips" to define $g_{c}^{(1)}$. In both basins, the techniques of Sect. 3.2 are applied to construct the quasi-Newton mapping $g_{c}$. It is straightened to $f_{d}$, and a homeomorphism is obtained by $h_{0}(c):=d$. It is 
permuting little "almonds," respecting their decomposition into four colors. Similar constructions are possible when one or both of the adjacent components of basins at $\mathcal{E}_{c}$ are not immediate, i.e. when the hyperbolic components at $\mathcal{E}_{M}$ are of greater depth [14]. Cf. $h_{1}, h_{2}$ in Figs. 6-7.
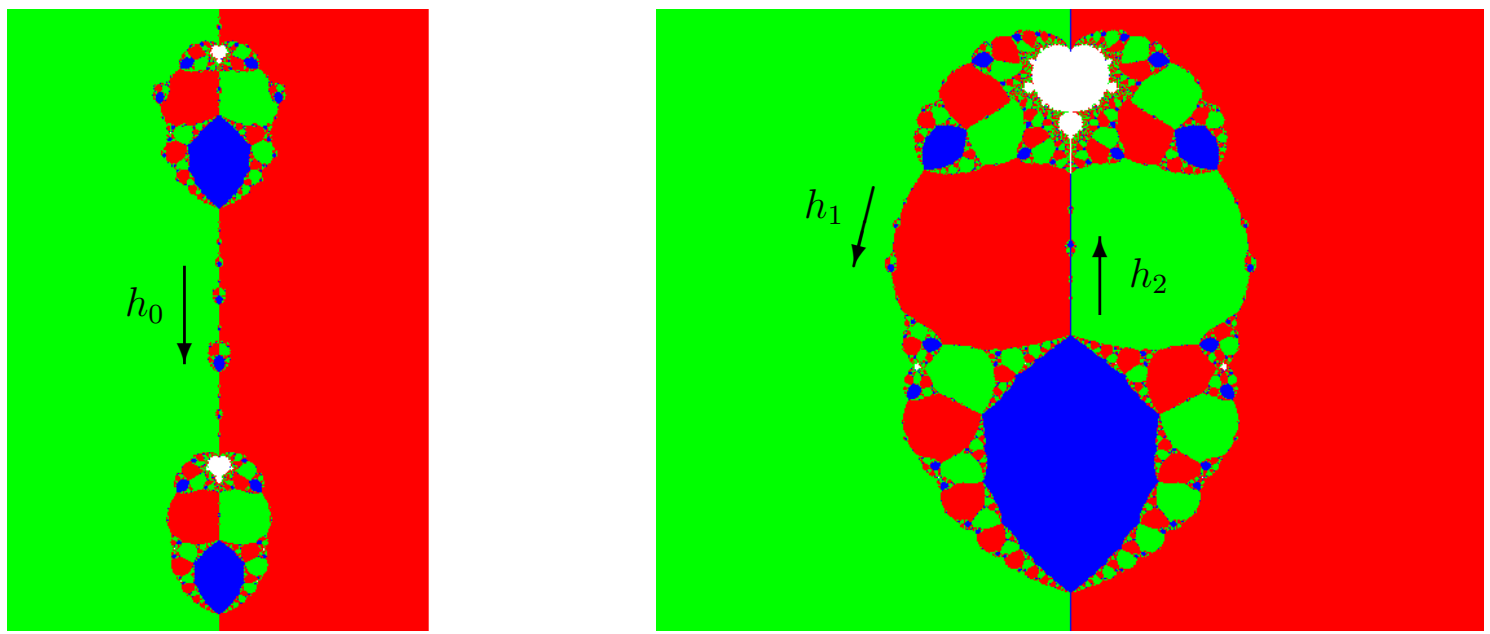

Figure 6: Homeomorphisms in the parameter space of Newton methods for cubic polynomials. Left: an "edge" between the "almonds" of orders 3 and 2. Right: homeomorphisms on edges within the almond of order 2. (The different colors, or shades of gray, indicate that the free critical point is attracted to one of the roots of the corresponding polynomial.)
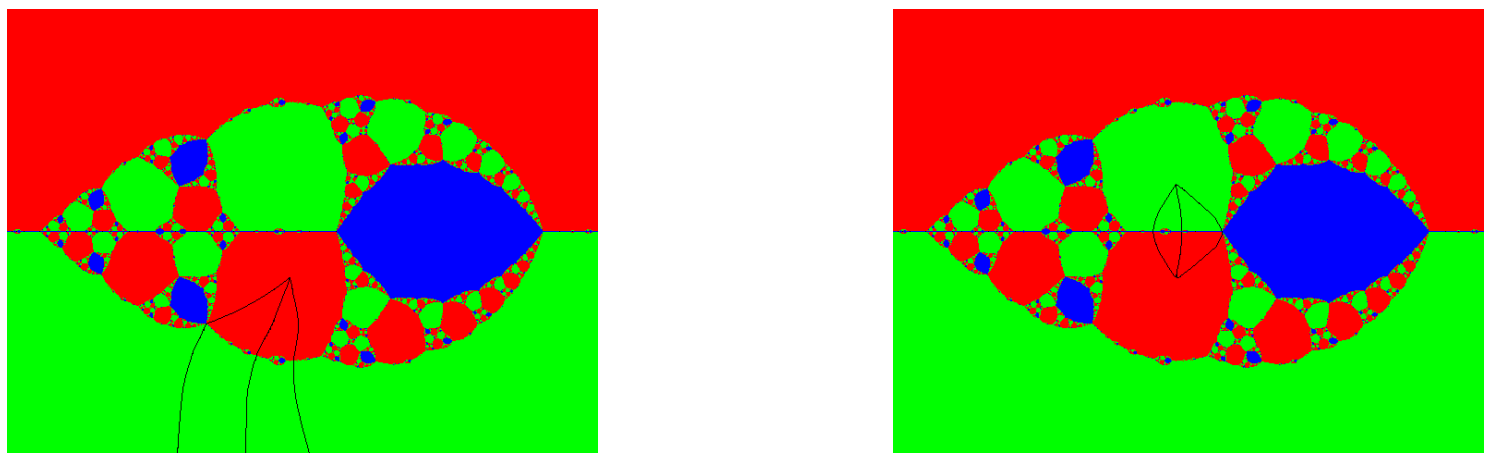

Figure 7: Cutting the Julia set with dynamic rays belonging to two different basins, to define the strips $V_{c}, W_{c}$ and the mapping $g_{c}^{(1)}$. This yields the homeomorphisms $h_{1}$ (left) and $h_{2}$ (right) in the almond of order 2 (cf. Fig. 6).

Cubic Newton methods are understood as matings of cubic polynomials [18], and there are analogous homeomorphisms in the parameter space of cubic polynomials with one superattracting fixed point. Again, the rays used in the piecewise definition of $g_{c}^{(1)}$ belong to the basins of two attracting fixed points, but one is finite and one at $\infty$ in the polynomial case. H. Hubbard has suggested to look at quadratic rational mappings with a superattracting cycle, which contain matings of quadratic polynomials. When we try to transfer a known homeomorphism of the Mandelbrot set to this family, in general we will have to use articulated rays to cut the Julia set. 
Although it may be possible to define $g_{c}^{(1)}$, it will not be possible to construct the quasi-regular mapping $g_{c}$, because the shift discontinuity happens not only within the basin of attraction, but at pinching points of the Julia set as well. For the same reason, it will not be possible to transfer a homeomorphism of $\mathcal{M}$ to a neighborhood of a copy of $\mathcal{M}$ in the cubic Newton family.

\section{Homeomorphism Groups of $\mathcal{M}$}

Denote the group of orientation-preserving homeomorphisms $h: \mathcal{M} \rightarrow \mathcal{M}$ by $\mathcal{G}_{M}$. If two homeomorphisms coincide on $\partial \mathcal{M}$, they encode the same information on the topological structure of $\mathcal{M}$. To avoid trivialities, we suggest some definitions of groups of non-trivial homeomorphisms as well.

\section{Definition 5.1 (Groups of Homeomorphisms)}

1. $\mathcal{G}_{M}$ is the group of homeomorphisms $h: \mathcal{M} \rightarrow \mathcal{M}$ that are orientation-preserving at branch points, and orientation-preserving in the interior of $\mathcal{M}$.

2. $\mathcal{G}_{a}$ is the group of homeomorphisms $h: \mathcal{M} \rightarrow \mathcal{M}$ that are orientation-preserving at branch points, and analytic in the interior of $\mathcal{M}$.

3. $\mathcal{G}_{b}$ is the group of homeomorphisms $h: \partial \mathcal{M} \rightarrow \partial \mathcal{M}$ that are orientation-preserving at branch points, and orientation-preserving on the boundaries of hyperbolic components.

4. $\mathcal{G}_{q}$ is the factor group $\mathcal{G}_{M} / \mathcal{G}_{1}$, where $\mathcal{G}_{1}$ is the normal subgroup consisting of trivial homeomorphisms: $\mathcal{G}_{1}:=\left\{h \in \mathcal{G}_{M} \mid h=\mathrm{id}\right.$ on $\left.\partial \mathcal{M}\right\}$.

$\mathcal{G}_{q}$ is the most natural definition of non-trivial homeomorphisms. $\mathcal{G}_{a}, \mathcal{G}_{b}, \mathcal{G}_{q}$ may well turn out to be mutually isomorphic. On $\mathcal{G}_{M}, \mathcal{G}_{a}$, and $\mathcal{G}_{b}$, define a metric by

$$
\begin{aligned}
d\left(h_{1}, h_{2}\right) & :=\left\|h_{1}-h_{2}\right\|_{\infty}+\left\|h_{1}^{-1}-h_{2}^{-1}\right\|_{\infty} \\
& :=\max \left|h_{1}(c)-h_{2}(c)\right|+\max \left|h_{1}^{-1}(c)-h_{2}^{-1}(c)\right|,
\end{aligned}
$$

where the maxima are taken over $c \in \mathcal{M}$ or $c \in \partial \mathcal{M}$, respectively. $\mathcal{G}_{q}$ consists of equivalence classes of homeomorphisms coinciding on the boundary, $[h]=h \mathcal{G}_{1}=$ $\mathcal{G}_{1} h$. Since $\mathcal{G}_{1}$ is closed, a metric is given by

$$
\begin{aligned}
d\left(\left[h_{1}\right],\left[h_{2}\right]\right) & :=\inf \left\{\left\|h_{1}^{\prime}-h_{2}^{\prime}\right\|_{\infty} \mid h_{1}^{\prime} \in\left[h_{1}\right], h_{2}^{\prime} \in\left[h_{2}\right]\right\} \\
& +\inf \left\{\left\|h_{1}^{\prime-1}-h_{2}^{\prime-1}\right\|_{\infty} \mid h_{1}^{\prime} \in\left[h_{1}\right], h_{2}^{\prime} \in\left[h_{2}\right]\right\} \\
& =\inf \left\{\left\|h_{1} \circ u-h_{2}\right\|_{\infty}+\left\|h_{1}^{-1} \circ v-h_{2}^{-1}\right\|_{\infty} \mid u, v \in \mathcal{G}_{1}\right\} .
\end{aligned}
$$

It may be more natural to take the infimum of a sum instead of the sum of infima in (5), i.e. inf $d\left(h_{1}^{\prime}, h_{2}^{\prime}\right)$, but I do not know how to prove the triangle inequality in that case. (6) is obtained from (5) by employing the facts that $\mathcal{G}_{1}$ is normal, and that right translations are isometries of the norm: $\left\|h_{1}-h_{2}\right\|_{\infty}=\left\|h_{1} \circ h-h_{2} \circ h\right\|_{\infty}$, since $h \in \mathcal{G}_{M}$ is bijective. 


\section{Proposition 5.2 (Topology of Homeomorphisms Groups)}

1. $\mathcal{G}_{M}, \mathcal{G}_{a}, \mathcal{G}_{b}, \mathcal{G}_{q}$ are complete metric spaces and topological groups, i.e. composition and inversion are continuous.

2. For $\mathcal{G}=\mathcal{G}_{M}, \mathcal{G}_{a}, \mathcal{G}_{b}, \mathcal{G}_{q}$ we have: if $\Omega_{1}, \Omega_{2}$ are hyperbolic components, then $\mathcal{N}:=\left\{h \in \mathcal{G} \mid h\left(\partial \Omega_{1}\right)=\partial \Omega_{2}\right\}$ is open.

Proof: 1. For $\mathcal{G}_{M}, \mathcal{G}_{a}, \mathcal{G}_{b}$, the proof is straightforward. But suppose we had used the alternative metric $\widetilde{d}\left(h_{1}, h_{2}\right):=\left\|h_{1}-h_{2}\right\|_{\infty}$, and $\left(h_{n}\right) \subset \mathcal{G}_{M}$ is a Cauchy sequence in that metric. Then it is converging uniformly to a continuous, surjective $h: \mathcal{E}_{M} \rightarrow \mathcal{E}_{M}$. If $h$ is injective, then $h_{n}^{-1} \rightarrow h^{-1}$ uniformly. But $h$ need not be injective, a counterexample is constructed in item 2 of [8, Prop. 7.7]. Thus, if we had used $\tilde{d}$ instead of $d$, the topology of $\mathcal{G}_{M}, \mathcal{G}_{a}, \mathcal{G}_{b}$ would be the same, but they would be incomplete metric spaces.

Now suppose $\left(\left[h_{n}\right]\right)$ is a Cauchy sequence in $\mathcal{G}_{q}$. It is sufficient to show that a subsequence converges, and without restriction we have $d\left(\left[h_{n+1}\right],\left[h_{n}\right]\right) \leq 3^{-n}$. Choose $u_{n}, v_{n} \in \mathcal{G}_{1}$ with

$$
\left\|h_{n+1} \circ u_{n}-h_{n}\right\|_{\infty} \leq 2^{-n} \text { and }\left\|h_{n+1}^{-1} \circ v_{n}-h_{n}^{-1}\right\|_{\infty} \leq 2^{-n} .
$$

Define the sequences

$$
\widehat{h}_{n}:=h_{n} \circ u_{n-1} \circ u_{n-2} \circ \ldots \circ u_{1} \text { and } \tilde{h}_{n}:=h_{n}^{-1} \circ v_{n-1} \circ v_{n-2} \circ \ldots \circ v_{1} \text {. }
$$

Since the maximum norm is invariant under right translations on $\mathcal{M}$, they satisfy

$$
\left\|\widehat{h}_{n+1}-\widehat{h}_{n}\right\|_{\infty} \leq 2^{-n} \text { and }\left\|\widetilde{h}_{n+1}-\widetilde{h}_{n}\right\|_{\infty} \leq 2^{-n}
$$

and there are continuous functions $\widehat{h}, \widetilde{h}$ with $\widehat{h}_{n} \rightarrow \widehat{h}$ and $\widetilde{h}_{n} \rightarrow \widetilde{h}$ uniformly. Now $\widehat{h} \circ \widetilde{h}$ and $\widetilde{h} \circ \widehat{h}$ are uniform limits of a sequence in $\mathcal{G}_{1}$, thus surjective, and $\widehat{h}$ is a homeomorphism. We have $\widehat{h}_{n} \rightarrow \widehat{h}$ in $\mathcal{G}_{M}$ and $\left[h_{n}\right]=\left[\widehat{h}_{n}\right] \rightarrow[\widehat{h}]$ in $\mathcal{G}_{q}$, therefore $\mathcal{G}_{q}$ is complete.

2. Hyperbolic components can be distinguished topologically from non-hyperbolic components, since only the boundary of a hyperbolic component contains a countable dense set of pinching points (by the Branch Theorem [15]). Thus every homeomorphism of $\mathcal{M}$ or $\partial \mathcal{M}$ is permuting the set of hyperbolic components or of their boundaries, respectively. Fix $a, b \in \partial \Omega_{2}$, and choose $\varepsilon>0$ such that no hyperbolic component $\neq \Omega_{2}$ is meeting both of the disks of radius $\varepsilon$ around $a$ and $b$. This is possible, since there are several external rays landing at $\partial \Omega_{2}$. If $h_{0} \in \mathcal{N}$ and $h \in \mathcal{G}$ with $d\left(h, h_{0}\right)<\varepsilon$, then $\left|h\left(h_{0}^{-1}(a)\right)-a\right|<\varepsilon$ and $\left|h\left(h_{0}^{-1}(b)\right)-b\right|<\varepsilon$, thus $h\left(\partial \Omega_{1}\right)=\partial \Omega_{2}$. (Analogously for the classes in $\left.\mathcal{G}_{q}.\right)$

\section{Theorem 5.3 (Groups of Non-Trivial Homeomorphisms)}

The groups of non-trivial homeomorphisms of $\mathcal{M}$ or $\partial \mathcal{M}-\mathcal{G}_{a}, \mathcal{G}_{b}$ and $\mathcal{G}_{q}-$ share the following properties:

1. They have the cardinality of the continuum $\mathbb{R}$, and they are totally disconnected. 
2. They are perfect, and not compact (not even locally compact).

3. A family of homeomorphisms $\mathcal{F} \subset \mathcal{G}_{a}, \mathcal{G}_{b}, \mathcal{G}_{q}$ is called normal, if its closure is sequentially compact. A necessary condition is that for every hyperbolic component $\Omega \subset \mathcal{M}$, there are only finitely many components of the form $h^{ \pm 1}(\Omega), h \in \mathcal{F}$. If $\mathcal{M}$ is locally connected, this condition will be sufficient for $\mathcal{F}$ being normal.

By composition, the homeomorphisms constructed by surgery according to Thm. 1.1 generate a countable subgroup of $\mathcal{G}_{a}, \mathcal{G}_{b}$ or $\mathcal{G}_{q}$. Will it be dense? - For $\mathcal{G}_{M}$, items 1 and 3 are wrong, and item 2 is true but trivial. Hence the motivation to consider the groups of non-trivial homeomorphisms. The same results hold for the analogous groups, where the condition of preserving the orientation is dropped.

Proof: We prove the statements for $\mathcal{G}_{a}$, the case of $\mathcal{G}_{b}$ or $\mathcal{G}_{q}$ is similar. There is a sequence of disjoint subsets $\mathcal{E}_{n} \subset \mathcal{M}$ with $\operatorname{diam}\left(\mathcal{E}_{n}\right) \rightarrow 0$, and a sequence of analytic homeomorphisms $h_{n}: \mathcal{M} \rightarrow \mathcal{M}$, such that $h_{n}=$ id on $\mathcal{M} \backslash \mathcal{E}_{n}, h_{n} \neq$ id. To construct these, fix a homeomorphism $h_{*}: \mathcal{E}_{M} \rightarrow \mathcal{E}_{M}$ according to Thm. 1.1, e.g. that of Figs. 2 and 3. Choose $\mathcal{E}_{0} \subset \mathcal{E}_{M}$ and a homeomorphism $h_{0}: \mathcal{E}_{0} \rightarrow \mathcal{E}_{0}, h_{0} \neq \mathrm{id}$, such that $\mathcal{E}_{0}$ is contained in a fundamental domain of $h_{*}$. This is possible e.g. by the tuning construction from Sect. 4.4. Then set $h_{n}:=h_{*}^{n} \circ h_{0} \circ h_{*}^{-n}$ on $\mathcal{E}_{n}:=h_{*}^{n}\left(\mathcal{E}_{0}\right)$, and extend it by the identity to a homeomorphism of $\mathcal{M}$. We have $\operatorname{diam}\left(\mathcal{E}_{n}\right) \rightarrow 0$ by the scaling properties of $\mathcal{M}$ at Misiurewicz points [17]. - An alternative approach is as follows: construct homeomorphisms $h_{n}: \mathcal{E}_{n} \rightarrow \mathcal{E}_{n}$, such that $\mathcal{E}_{n}$ is contained in the $\operatorname{limb} \mathcal{M}_{1 / n}$, then $\operatorname{diam}\left(\mathcal{E}_{n}\right) \rightarrow 0$ by the Yoccoz inequality. These homeomorphisms can be constructed by tuning, or at $\beta$-type Misiurewicz points according to Sect. 4.2, or on edges (Sect. 4.3). All of the homeomorphisms constructed below extend to homeomorphisms of $\mathbb{C}$, cf. item 3 of Remark 3.2. (If $\mathcal{M}$ is locally connected, all homeomorphisms in $\mathcal{G}_{M}, \mathcal{G}_{a}$ or $\mathcal{G}_{b}$ extend to homeomorphisms of $\mathbb{C}$.)

1. We construct an injection $(0,1) \rightarrow \mathcal{G}_{a}, x \mapsto h$ as follows: expand $x$ in binary digits (not ending on $\overline{1}$ ). Set $h:=h_{n}$ or $h:=$ id on $\mathcal{E}_{n}$, if the $n$-th digit is 1 or 0 , respectively, and $h:=$ id on $\mathcal{M} \backslash \cup \mathcal{E}_{n}$. Although the sequence of sets $\mathcal{E}_{n}$ will accumulate somewhere, continuity of $h$ can be shown by employing $\operatorname{diam}\left(\mathcal{E}_{n}\right) \rightarrow 0$. - Conversely, to obtain an injection $\mathcal{G}_{a} \rightarrow(0,1), h \mapsto x$, enumerate the hyperbolic components $\left(\Omega_{n}\right)_{n \in \mathbb{N}}$, and denote the $n$-th prime number by $p_{n}$. Now $x$ shall have the digit 1 at the place $p_{n}^{m}$, iff $h: \Omega_{n} \rightarrow \Omega_{m}$. The mapping $h \mapsto x$ is injective, since the homomorphism from $\mathcal{G}_{a}$ to the permutation group of hyperbolic components is injective: if $h$ is mapping every hyperbolic component to itself, it is fixing the points of intersection of closures of hyperbolic components, i.e. all roots of satellite components. These are dense in $\partial \mathcal{M}$, thus $h=\mathrm{id}$. By the two injections, $\left|\mathcal{G}_{a}\right|=|(0,1)|=|\mathbb{R}|$.

If $h_{1}, h_{2} \in \mathcal{G}_{a}$ with $h_{1} \neq h_{2}$, there is a hyperbolic component $\Omega$ with $h_{1}(\Omega) \neq h_{2}(\Omega)$. By Prop. 5.2, $\mathcal{N}:=\left\{h \in \mathcal{G}_{a} \mid h(\Omega)=h_{1}(\Omega)\right\}$ is an open neighborhood of $h_{1}$, and $\mathcal{G}_{a} \backslash \mathcal{N}=\bigcup\left\{h \in \mathcal{G}_{a} \mid h(\Omega)=\Omega^{\prime}\right\}$ is an open neighborhood of $h_{2}$, where the union is taken over all hyperbolic components $\Omega^{\prime} \neq h_{1}(\Omega)$. Thus $h_{1}$ and $h_{2}$ belong to 
different connected components, and $\mathcal{G}_{a}$ is totally disconnected.

2. We have $d\left(h_{n}, \mathrm{id}\right) \leq 2 \operatorname{diam}\left(\mathcal{E}_{n}\right) \rightarrow 0$ as $n \rightarrow \infty$, thus id is not isolated in $\mathcal{G}_{a}$. Since composition is continuous, no point is isolated, and $\mathcal{G}_{a}$ is perfect.

Choose a homeomorphism $h: \mathcal{E}_{M} \rightarrow \mathcal{E}_{M}$ according to Thm. 1.1, which is expanding at $a$ and contracting at $b$, extend it by the identity to $h \in \mathcal{G}_{a}$. The iterates of $h$ satisfy $h^{k}(a)=a$ and $h^{k}(c) \rightarrow b$ for all $c \in \mathcal{E}_{M} \backslash\{a\}$, thus the pointwise limit of $\left(h^{k}\right)_{k \in \mathbb{N}}$ is not continuous. The sequence does not contain a subsequence converging uniformly, and $\mathcal{G}_{a}$ is not sequentially compact, a fortiori not compact. - If $\mathcal{N}$ is a neighborhood of id in $\mathcal{G}_{a}$, fix an $n$ such that $\mathcal{N}$ contains the ball of radius $2 \operatorname{diam}\left(\mathcal{E}_{n}\right)$ around id, then $\mathcal{N}$ contains the sequence $\left(h_{n}^{k}\right)_{k \in \mathbb{N}}$. Thus $\mathcal{N}$ is not compact, and $\mathcal{G}_{a}$ is not locally compact.

3. When $\mathcal{F}$ does not satisfy the finiteness condition, there is a sequence $\left(h_{n}\right) \subset \mathcal{F}$ and a hyperbolic component $\Omega$, such that the period of $h_{n}(\Omega)$ (or $h_{n}^{-1}(\Omega)$ ) diverges. Assume $h_{n} \rightarrow h$, then $h_{n}(\Omega)=h(\Omega)$ for $n \geq N_{0}$ according to Prop. 5.2, a contradiction. If $\mathcal{F}$ satisfies the finiteness condition, a diagonal procedure yields a subsequence which is eventually constant on every hyperbolic component, thus respecting the partial order of hyperbolic components. Assuming local connectivity, all fibers are trivial [15], and $\lim h_{n}$ is obtained analogously to [8, Sect. 9.3].

Two rational angles with odd denominators are Lavaurs-equivalent, if the corresponding parameter rays are landing at the same root. Denote the closure of this equivalence relation on $S^{1}$ by $\sim$. The abstract Mandelbrot set is the quotient space $S^{1} / \sim[9]$, it is a combinatorial model for $\partial \mathcal{M}$, which will be homeomorphic to $\partial \mathcal{M}$ if $\mathcal{M}$ is locally connected. (It is analogous to Douady's pinched disk model of $\mathcal{M}$.) Orientation-preserving homeomorphisms of the abstract Mandelbrot set are described by orientation-preserving homeomorphisms $\mathbf{H}: S^{1} \rightarrow S^{1}$ that are compatible with $\sim$. According to Sect. 4.1, every homeomorphism $h: \mathcal{E}_{M} \rightarrow \mathcal{E}_{M}$ constructed by surgery defines such a circle homeomorphism (extended by the identity), and the homeomorphism group of $S^{1} / \sim$ has the properties given in Thm. 5.3. In fact, these homeomorphisms of the abstract Mandelbrot set can be constructed in a purely combinatorial way, without using quasi-conformal surgery [8].

\section{References}

[1] B. Branner, A. Douady, Surgery on complex polynomials, in: Holomorphic dynamics, Proc. 2nd Int. Colloq. Dyn. Syst., Mexico City, LNM 1345, 11-72 (1988).

[2] B. Branner, N. Fagella, Homeomorphisms between limbs of the Mandelbrot set, J. Geom. Anal. 9, 327-390 (1999).

[3] B. Branner, N. Fagella, Extensions of Homeomorphisms between Limbs of the Mandelbrot Set, Conform. Geom. Dyn. 5, 100-139 (2001). 
[4] X. Buff, C. Henriksen, Julia sets in parameter spaces, Comm. Math. Phys. 220, 333-375 (2001).

[5] L. Carleson, T. W. Gamelin, Complex dynamics, Springer, New York, 1993.

[6] A. Douady, J. H. Hubbard, On the dynamics of polynomial-like mappings, Ann. Sci. École Norm. Sup. 18, 287-343 (1985).

[7] P. Haïssinsky, Modulation dans l'ensemble de Mandelbrot, in: The Mandelbrot Set, Theme and Variations, Tan L. ed., LMS Lecture Notes 274, Cambridge University Press 2000.

[8] W. Jung, Homeomorphisms on Edges of the Mandelbrot Set, Ph.D. thesis RWTH Aachen 2002.

[9] K. Keller, Invariant Factors, Julia Equivalences and the (Abstract) Mandelbrot Set, LNM 1732, Springer 2000.

[10] O. Lehto, K. I. Virtanen, Quasiconformal Mappings in the Plane, Springer 1973.

[11] C. T. McMullen, The Mandelbrot set is universal, in: The Mandelbrot Set, Theme and Variations, Tan L. ed., LMS Lecture Notes 274, Cambridge University Press 2000 .

[12] J. Riedl, D. Schleicher, On Crossed Renormalization of Quadratic Polynomials, in: Proceedings of the 1997 conference on holomorphic dynamics, RIMS Kokyuroku 1042, 11-31, Kyoto 1998.

[13] J. Riedl, Arcs in Multibrot Sets, Locally Connected Julia Sets and Their Construction by Quasiconformal Surgery, Ph.D. thesis TU München 2000.

[14] P. Roesch, Topologie locale des methodés de Newton cubiques, Ph.D. thesis ENS de Lyon 1997.

[15] D. Schleicher, On Fibers and Local Connectivity of Mandelbrot and Multibrot Sets, IMS-preprint 98-13a (1998).

[16] D. Schleicher, Rational Parameter Rays of the Mandelbrot Set, Astérisque 261, 405443 (2000).

[17] Tan L., Similarity between the Mandelbrot set and Julia sets, Comm. Math. Phys. 134, 587-617 (1990).

[18] Tan L., Branched coverings and cubic Newton maps, Fundam. Math. 154, 207-260 (1997).

Present address: Wolf Jung, Inst. Reine Angew. Math.

RWTH Aachen, D-52056 Aachen, Germany

http://www.iram.rwth-aachen.de/ jung

jung@iram.rwth-aachen.de 